\title{
COMPLETE CHARACTERIZATION OF FUNCTIONS WHICH ACT, VIA SUPERPOSITION, ON SOBOLEV SPACES
}

BY

MOSHE MARCUS $^{1}$ AND VICTOR J. MIZEL ${ }^{2}$

\begin{abstract}
Given a domain $\boldsymbol{\Omega} \subset \boldsymbol{R}_{N}$ and a Borel function $h: \boldsymbol{R}_{m} \rightarrow \boldsymbol{R}$, conditions on $h$ are sought ensuring that for every $m$-tuple of functions $u_{i}$ belonging to the first order Sobolev space $W^{1} P(\Omega)$, the function $h\left(u_{1}(\cdot), \ldots, u_{m}(\cdot)\right)$ will belong to a first order Sobolev space $W^{1, r}(\Omega)$, $1<r<p<\infty$. In this paper conditions are found which are both necessary and sufficient in order that $h$ have the above property. This result is based on a characterization obtained here for those Borel functions $g$ : $\boldsymbol{R}_{m} \times\left(\boldsymbol{R}_{N}\right)_{m} \rightarrow \boldsymbol{R}$ satisfying the requirement that for every $m$-tuple of functions $u_{i} \in W^{1, p}(\Omega)$ the function $g\left(u_{1}(\cdot), \ldots, u_{m}(\cdot), \nabla u_{1}(\cdot)\right.$, $\left.\ldots, \nabla u_{m}(\cdot)\right)$ belongs to $L^{r}(\Omega)$. A needed result on the measurability of the set of $\boldsymbol{R}_{k}$-Lebesgue points of a function on $\boldsymbol{R}_{N}$ is presented in an appendix.
\end{abstract}

1. Introduction. Let $\Omega$ be a domain in $R_{N}$ and let $\Re(\Omega)$ denote the space of real measurable functions in $\Omega$. Given a Borel function $h: R_{m} \rightarrow R$ we define the superposition mapping $T_{h}: \mathfrak{N}(\Omega) \rightarrow \mathfrak{N}(\Omega)$ by

$$
T_{h} \mathbf{u}=h \circ \mathbf{u}, \quad \mathbf{u}=\left(u_{1}, \ldots, u_{m}\right) \in \mathscr{N}(\Omega)^{m} .
$$

Let $W_{1, p}(\Omega), 1 \leqslant p \leqslant \infty$, denote the space of functions in $L_{p}(\Omega)$ whose first order distribution derivatives belong to $L_{p}(\Omega)$. The standard norm in this space is

$$
\|u\|_{W_{1, p}(\Omega)}=\|u\|_{L_{p}(\Omega)}+\sum_{q=1}^{N}\left\|D_{i} u\right\|_{L_{p}(\Omega)}, \quad u \in W_{1, p}(\Omega),
$$

where $D_{1} u, \ldots, D_{N} u$ denote the first order distribution derivatives of $u$ with respect to some coordinate system in $R_{N}$.

Suppose that $\Omega$ is bounded and satisfies the cone condition. If $h$ is locally Lipschitz and if the first order partial derivatives of $h$ satisfy a.e. in $\boldsymbol{R}_{\boldsymbol{m}}$ polynomial growth conditions, then $T_{h}$ maps $W_{1, p}(\Omega)^{m}$ into $W_{1, r}(\Omega)$, where $1 \leqslant r \leqslant p<\infty$ and the relation between $p$ and $r$ depends on the order of

Received by the editors April 5, 1978.

AMS (MOS) subject classifications (1970). Primary 46E35, 47H15; Secondary 46J15.

Key words and phrases. Locally Lipschitz function, cone condition, $k$-dimensional Hausdorff measure, regular Lebesgue point.

${ }^{1}$ Research supported by a grant from the Israeli National Academy of Sciences.

${ }^{2}$ Research supported by National Science Foundation Grants MPS71-03776-A05 and MCS-77-03643. 
the polynomials in the growth conditions. Furthermore, this mapping is bounded. This result, in a somewhat different form, was obtained in [5]. For $m=1$ the result was previously obtained by Stampacchia [9] when $h$ is uniformly Lipschitz and by Serrin in the general case. Serrin's result also shows that $D_{i}\left(T_{h} u\right)$ can be computed by the standard chain rule. (For details see [4].)

The question whether the above mentioned conditions are also necessary in order that $T_{h}$ should map $W_{1, p}(\Omega)^{m}$ into $W_{1, r}(\Omega)$ proved to be considerably more difficult and it remained open for several years. The main purpose of the present paper is to provide an affirmative answer to this question. To achieve this result we first consider a related problem for superposition operators which map Sobolev spaces into Lebesgue spaces.

Let $g: R_{m} \times R_{m N} \rightarrow R$ be a Borel function and let $S_{g}: W_{1,1}^{\text {loc }}(\Omega)^{m} \rightarrow \mathfrak{T}(\Omega)$ be the mapping defined by

$$
S_{g} \mathrm{u}=g\left(u_{1}, \ldots, u_{m}, D_{1} u_{1}, \ldots, D_{N} u_{1}, \ldots, D_{1} u_{m}, \ldots, D_{N} u_{m}\right)
$$

where $\mathbf{u}=\left(u_{1}, \ldots, u_{m}\right)$. We show that if $S_{g}$ maps $W_{1, p}(\Omega)^{m}$ into $L_{r}(\Omega)$, $1<r<p<\infty$, then $g$ must satisfy certain polynomial growth conditions depending on $p$ and $r$. For instance, if $p<N$ the condition is

$$
|g(\xi, \eta)|<\operatorname{const}\left(1+|\xi|^{q / r}+|\eta|^{p / r}\right), \quad \text { with } q=N p /(N-p),
$$

for every $\eta$ in $R_{m N}$, for a.e. $\xi$ in $R_{m}$. This result leads almost directly to the necessity of the conditions on $h$ when $m=1$. This is due to the fact that, when $m=1$, the chain rule for $D_{i}\left(T_{h} u\right)$ is available. When $m>1$ the chain rule for $D_{i}\left(T_{h} u\right)$ does not hold in general. In this case the necessity of the conditions on $h$ is established by combining the result already proved for $m=1$ with the result concerning the mapping $S_{g}$ for $m>1$.

Results similar to those described above hold also in the case where $\Omega$ is unbounded. In this case the conditions are stronger than in the case of bounded domains.

The plan of the paper is as follows. In $\$ 2$ we state the main results in the case of bounded domains. $\$ 3$ and $\S 4$ (together with an appendix) are devoted to the development of auxiliary results. The main result for the mapping $S_{g}$ is proved in $\$ 5$ and for the mapping $T_{h}$ in $\$ 6$ and $\$ 7$. The parallel results in the case of unbounded domains are presented in $\$ 8$. Finally in $\$ 9$ and $\$ 10$ we discuss some additional results and open questions related to the mappings $S_{g}$ and $T_{h}$.

2. Statement of main results. In this section $\Omega$ denotes a domain in $R_{N}$ satisfying the cone condition. The generic points in $R_{N}, R_{m}$ and $R_{m N}$ will be denoted by $x=\left(x_{1}, \ldots, x_{n}\right), \xi=\left(\xi_{1}, \ldots, \xi_{m}\right)$ and $\eta=$ $\left(\eta_{1,1}, \ldots, \eta_{1, N}, \ldots, \eta_{m, 1}, \ldots, \eta_{m, N}\right)$ respectively. The norm $|\cdot|$ of such a point will always mean Euclidean norm. 
The first result concerns superposition mappings of the type (1.1).

Theorem 1. Suppose that $\Omega$ is bounded. Let $h: R_{m} \rightarrow R$ be a Borel function and let $p, r$ be two numbers such that $1<r<p<N$. Then $T_{h}$ maps $W_{1, p}(\Omega)^{m}$ into $W_{1, r}(\Omega)$ if and only if the following conditions hold:

(i) $h$ is locally Lipschitz in $R_{m}$.

(ii) The first order partial derivatives of $h$ satisfy the inequality

$$
\left|\frac{\partial h}{\partial \xi_{i}}(\xi)\right|<a_{0}\left(1+|\xi|^{\nu}\right) \quad \text { a.e. in } R_{m}, i=1, \ldots, m,
$$

where $a_{0}$ is a constant and $\nu=N(p-r) /(r(N-p))$.

If $N<p($ or $N=1$ and $1<p)$ and $1<r<p$ then $T_{h}$ maps $W_{1, p}(\Omega)^{m}$ into $W_{1, r}(\Omega)$ if and only if condition (i) holds.

In either case, if $T_{h}$ maps $W_{1, p}(\Omega)^{m}$ into $W_{1, r}(\Omega)$ then the mapping is bounded and the following inequalities hold:

$$
\begin{array}{ll}
\left\|T_{h} \mathbf{u}\right\|_{W_{1,(}(\Omega)} \leqslant b_{0}\left(1+\sum_{i=1}^{m}\left\|u_{i}\right\|_{W_{1,}(\Omega)}^{\nu+1}\right), & \text { if } p<N, \\
\left\|T_{h} \mathrm{u}\right\|_{W_{1, r}(\Omega)} \leqslant b(M)\left(1+\sum_{i=1}^{m}\left\|u_{i}\right\|_{W_{1, p}(\Omega)}\right), & \text { if } p>N(\text { or } N=1 \text { and } p>1) \\
& \text { and } \sum_{i=1}^{m}\left\|u_{i}\right\|_{W_{1,}(\Omega)}<M .
\end{array}
$$

The constants $b_{0}$ and $b(M)$ depend on $m, a_{0}, h(0)$ and $\Omega$. (Of course, $b(M)$ depends also on $M$.)

The second result concerns superposition mappings of the type (1.3).

Theorem 2. Suppose that $\Omega$ is bounded. Let $g: R_{m} \times R_{m N} \rightarrow R$ be a Borel function and let $p, r$ be two numbers such that $1<r<p<\infty$. Suppose that $S_{g}$ maps $W_{1, p}(\Omega)^{m}$ into $L_{r}(\Omega)$. Then $g$ satisfies the following conditions.

If $p<N$, there exists a constant $c_{0}$ such that

$$
|g(\xi, \eta)| \leqslant c_{0}\left(1+|\xi|^{q / r}+|\eta|^{p / r}\right), \quad \text { with } q=N p /(N-p),
$$

for every $\eta$ in $R_{m N}$ and a.e. $\xi$ in $R_{m}$. The exceptional null set in $R_{m}$ may vary with $\eta$.

If $p>N($ or $N=1$ and $p \geq 1)$, then for every $M>0$ there exists a constant $c(M)$ such that

$$
|g(\xi, \eta)| \leqslant c(M)\left(1+|\eta|^{p / r}\right)
$$

for every $\eta$ in $R_{m N}$ and a.e. $\xi$ in $R_{m}$ such that $|\xi|<M$. Again, the exceptional null set in $R_{m}$ may vary with $\eta$. 
If a function $g$ satisfies (2.3) or (2.4) everywhere in $R_{m} \times R_{m N}$, then (in view of the Sobolev imbedding theorem) $S_{g}$ maps $W_{1, p}(\Omega)^{m}$ into $L_{r}(\Omega)$ for the appropriate values of $p$ and $r$. Therefore if $g$ is continuous the conditions described in Theorem 2 are necessary and sufficient for $S_{g}$ to map $W_{1, p}(\Omega)^{m}$ into $L_{r}(\Omega)$. However the assumption of continuity can be replaced by various weaker assumptions. Results of this type will be discussed in $\$ 9$.

Results similar to those stated above hold also in the case where $\Omega$ is unbounded. These will be presented in $\$ 8$.

3. Construction of a class of elementary functions. The proof of Theorems 1 and 2 calls for the construction of functions in $W_{1, p}(\Omega)$ according to a given set of specifications. One of the requirements is that the gradient of the function should attain a prescribed sequence of values on sets of prescribed measure and at the same time the values of the function should lie within a given range in each of these sets. In the construction of these functions we use certain piecewise linear functions which we describe below.

LEMMA 1. Let $z \in R_{N}, z \neq 0$, and let $\sigma$ be a positive number. Then there exists a piecewise linear function $w$ in the cube $E_{\sigma}=\left\{x \in R_{N}:\left|x_{i}\right|<\sigma / 2\right.$, $i=1, \ldots, N\}$ such that,

(i) $w=0$ on $\partial E_{o}$,

(ii) $|\nabla w|$ is either zero or $|z|$ in $E_{\sigma}$,

(iii) $\nabla w=z$ in a cube $F$ contained in $E_{\sigma}$, whose sides are parallel to the axes and which satisfies

$$
\mu_{N}(F) / \sigma^{N} \geqslant a_{N}>0 \text {. }
$$

Here $\mu_{N}$ denotes $N$-dimensional Lebesgue measure and $a_{N}$ is a constant which depends only on $N$.

Proof. Let $b=\sigma / 2 \sqrt{N}$ and let $T$ be a cube in $R_{N}$ of side length $b$ such that one of its vertices (say $P$ ) lies in the half space $z \cdot x>0$, the $N$ vertices nearest to $P$ lie in the hyperplane $z \cdot x=0$ and one of these vertices is at the origin. Let $T^{\prime}=T \cap\{z \cdot x>0\}$ and define a function $w_{0}$ in $T$ as follows:

$$
w(x)=z \cdot x \quad \text { in } T^{\prime}, \quad w(x)=0 \quad \text { in } T \backslash T^{\prime} .
$$

Note that $\mu_{N}\left(T^{\prime}\right) / \mu_{N}(T)$ depends only on $N$.

Let $P^{\prime}$ be that vertex of $T$ for which $\overline{P P^{\prime}}$ is one of the main diagonals of $T$. Let $U$ be a closed cube of side $2 b$ such that $U \supset T$, the sides of $U$ are parallel to those of $T$ and $P^{\prime}$ is a vertex of $U$. We extend $w_{0}$ to $U$ by $N$ successive reflexions. In order to describe these reflexions we use a set of coordinates $\left(\bar{x}_{1}, \ldots, \bar{x}_{N}\right)$ whose origin is at $P^{\prime}$ and with respect to which $T=\{\bar{x}$ : $\left.0<\bar{x}_{i}<b, i=1, \ldots, N\right\}$. Let $T^{*}$ be the image of $T$ by reflexion with respect to $\bar{x}_{1}=b$ and set $T_{1}=T \cup T^{*}$. We extend $w_{0}$ to $T_{1}$ by reflexion with 
respect to $\bar{x}_{1}=b$. Let $k$ be an integer, $1 \leqslant k \leqslant N$, and set $T_{k}=\{\bar{x}$ : $0 \leqslant \bar{x}_{i} \leqslant 2 b$ for $i=1, \ldots, k$ and $0 \leqslant \bar{x}_{i} \leqslant b$ for $\left.i=k+1, \ldots, N\right\}$.

If $k<N$, denote by $T_{k}^{*}$ the image of $T_{k}$ by reflexion with respect to $\bar{x}_{k+1}=b$. Then $T_{k+1}=T_{k} \cup T_{k}^{*}$. Assuming that $w_{0}$ is defined in $T_{k}$ we extend it to $T_{k+1}$ by reflexion with respect to $\bar{x}_{k+1}=b$. Thus after $N$ steps we obtain an extension of $w_{0}$ to the entire cube $U$.

Next let $V$ be a cube whose sides are parallel to the axes $\left(x_{1}, \ldots, x_{N}\right)$, whose center is at the center of $U$ and whose side length is $2 \sqrt{N} b$. We extend $w_{0}$ to $V$ by setting $w_{0}=0$ in $V \backslash U$. Let $B$ be the largest ball contained in $T^{\prime}$ and let $F^{\prime}$ be the cube inscribed in $B$ whose sides are parallel to the axes. Then $\mu_{N}\left(F^{\prime}\right) / b^{N}$ depends only on $N$. Finally, let $x^{0}$ be the center of $V$ and set $w(x)=w_{0}\left(x+x^{0}\right)$ for every $x$ in $E_{\sigma}=-x_{0}+V$. Clearly the function $w$ satisfies all the assumptions of the lemma. In particular (iii) holds with respect to the cube $F=-x_{0}+F^{\prime}$.

We shall often use a variant of Lemma 1 which, for purposes of reference, we shall state separately.

Lemma 2. Let $z^{1}, \ldots, z^{m} \in R_{N}, z^{i} \neq 0(i=1, \ldots, m)$, and let $\sigma$ be $a$ positive number. Then there exist piecewise linear functions $w_{1}, \ldots, w_{m}$ in $E_{o}$ such that $w_{i}$ satisfies the conditions of Lemma 1 with $z=z^{i}$ but with $F$ independent of $i$ such that

$$
\mu_{N}(F) / \sigma^{N} \geqslant a_{N}^{m} .
$$

Proof. Let $w_{1}$ be as in Lemma 1 with $z=z^{1}$. Let $F_{1}$ be the cube mentioned in (iii) where $\nabla w_{1}=z^{1}$. Let $x^{1}$ be the center of $F_{1}$ and $\sigma_{1}$ its side length. Denote by $\tilde{w}_{2}$ the function described in Lemma 1 when $\sigma=\sigma_{1}$ and $z=z^{2}$, and define $w_{2}$ in $E_{\sigma}$ as follows:

$$
w_{2}(x)=\tilde{w}_{2}\left(x-x^{1}\right) \quad \forall x \in F_{1}, \quad w_{2}(x)=0 \quad \forall x \in E_{\sigma} \backslash F .
$$

Proceeding in this manner we obtain functions $w_{1}, \ldots, w_{m}$ and cubes $F_{1}, \ldots, F_{m}$ such that $w_{i}$ satisfies conditions (i)-(iii) of Lemma 1 with $z$ and $F$ replaced by $z^{i}$ and $F_{i}$. Moreover, $F_{1} \supset F_{2} \supset \ldots \supset F_{m}$, and $\mu_{N}\left(F_{1}\right) / \sigma^{N}>a_{n}$ and $\mu_{N}\left(F_{i+1}\right) / \mu_{N}\left(F_{i}\right) \geqslant a_{N}$ for $i=1, \ldots, m-1$. Thus the lemma holds with $F=F_{m}$.

REMARK. In the set $F$ the functions $w_{i}, i=1, \ldots, m$, are of the form $z^{i} \cdot\left(x-y^{i}\right)$ where $\left|y^{i}\right|<2 \sqrt{N}$ o. Thus

$$
\left\{\begin{array}{l}
w_{i}(x)=\sum_{n=1}^{N} z_{n}^{i} x_{n}+b_{i} \quad \forall x \in F, \text { and } \\
\left|b_{i}\right| \leqslant 2 \sqrt{N} \sigma\left|z^{i}\right| .
\end{array}\right.
$$


4. Auxiliary results. Let $g: R_{m} \times R_{m N} \rightarrow[0, \infty)$ be a Borel function. As before, we denote by $S_{g}$ the mapping from $W_{1, p}(\Omega)^{m}$ into $\Re(\Omega)$ given by

$$
\left(u_{1}, \ldots, u_{m}\right) \rightarrow g\left(u_{1}, \ldots, u_{m}, \nabla u_{1}, \ldots, \nabla u_{m}\right) \text {. }
$$

Here, as before, $\Omega$ is a domain in $R_{N}$.

With every point $\eta \in R_{m N}, \eta=\left(\eta_{1,1}, \ldots, \eta_{1, N}, \ldots, \eta_{m, 1}, \ldots, \eta_{m, N}\right)$, we associate the $m \times N$ matrix

$$
A(\eta)=\left(\eta_{i, j}\right)_{i=1, \ldots, m ; j=1, \ldots, N}
$$

The rank of this matrix will be denoted by $k(\eta)$. The image of $R_{N}$ by the linear transformation $x \rightarrow A(\eta) x$ will be denoted by $M_{\eta}$. Thus $M_{\eta}$ is a subspace of $R_{m}$ of dimension $k(\eta)$.

Suppose that $S_{g}$ maps $W_{1, p}(\Omega)^{m}$ into $L_{1}^{\text {loc }}(\Omega)$. Then, for every $\eta$ in $R_{m N}$ and every $\xi^{0}$ in $R_{m}$, the function $f\left(\cdot ; \xi^{0}, \eta\right): R_{N} \rightarrow R$ given by

$$
f\left(x ; \xi^{0}, \eta\right)=g\left(A(\eta) x+\xi^{0}, \eta\right) \quad \forall x \in R_{N}
$$

is locally integrable in $R_{N}$. Using this fact we shall show that for every $\eta$ in $R_{m N}$, the function $\xi \rightarrow g(\xi, \eta)$ is locally integrable in $\xi^{0}+M_{\eta}$ (with respect to $k(\eta)$-dimensional Hausdorff measure) for every fixed $\xi^{0}$ in $\boldsymbol{R}_{\boldsymbol{m}}$.

Let us assume (as we may) that the first $k(\eta)$ columns of $A(\eta)$ are linearly independent. We assume of course that $\eta \neq 0$ so that $k(\eta)>0$. Set

$$
B(\eta)=\left(\eta_{i, j}\right)_{i=1, \ldots, m ; j=1, \ldots, k}
$$

where $k=k(\eta)$. If $k<N$ and $\zeta \in R_{N-k}$ set

$$
\pi_{k}(\zeta)=\left\{x \in R_{N}: x_{k+1}=\zeta_{1}, \ldots, x_{N}=\zeta_{N-k}\right\} .
$$

If $k=N$ set $\pi_{k}=\pi_{k}(0)=R_{N}$. Clearly, the transformation $x \rightarrow A(\eta) x$ maps $\pi_{k}(\zeta)$ onto $M_{\eta}$ in a (1-1) manner.

Now, let $\xi^{0}$ be a point in $R_{m}$ and denote by $U_{0}(\cdot)=U\left(\cdot ; \xi^{0}, \eta\right)$ the mapping given by $U_{0}(x)=A(\eta) x+\xi^{0}, x \in R_{N}$. Let $\zeta$ be a point in $R_{N-k}$ such that $f\left(\cdot ; \xi^{0}, \eta\right)$ restricted to $\pi_{k}(\zeta)$ is locally integrable with respect to $\mu_{k}$ ( $=k$-dimensional Lebesgue measure). Let $E$ be a $\mu_{k}$-measurable set in $\pi_{k}(\zeta)$. Then by the change of variables formula for integration (see e.g. [1, p. 244]) we have

$$
\int_{U_{0}(E)} g(\xi, \eta) d \mathcal{H}_{k}(\xi)=\int_{E} g\left(A(\eta) x+\xi^{0}, \eta\right) J_{k}(B(\eta)) d \mu_{k}(x),
$$

where $\mathcal{H}_{k}$ denotes $k$-dimensional Hausdroff measure and $J_{k}(B(\eta))$ is the square root of the sum of the squares of the $k \times k$ minors of $B(\eta)$. By our choice of $\zeta$ the right-hand side of (4.2) is finite for every bounded $E$. Therefore $g(\cdot, \eta)$ is locally integrable with respect to $\mathcal{K}_{k}$ in $\xi^{0}+M_{\eta}$.

From the last assertion we deduce (again using $(4.2)$ ) that $f\left(\cdot ; \xi^{0}, \eta\right)$ restricted to $\pi_{k}(\zeta)$ is locally integrable for every $\zeta$ in $R_{N-k}$. 
Given $\eta \neq 0$ in $R_{m N}$ we denote by $P(\eta)$ the set of points $\{\xi\}$ in $R_{m}$ such that $\xi$ is a (regular) Lebesgue point of the function $g(\cdot, \eta)$ restricted to $\xi+M_{\eta}$, with respect to the measure $\mathcal{H}_{k(\eta)}$. Some properties of $P(\eta)$ will be needed in the next section. These properties are the subject of the following two lemmas.

LEMMA 3. $P(\eta)$ is a set of total measure in $R_{m}$, i.e. its complement is a Lebesgue null set.

LeMMA 4. Let $\xi^{\prime} \in P(\eta)$. Let $\xi^{0}$ and $x^{\prime}$ be points in $R_{m}$ and $R_{N}$ respectively such that

$$
\xi^{\prime}=A(\eta) x^{\prime}+\xi^{0}
$$

Then $x^{\prime}$ is a Lebesgue point of $f\left(\cdot ; \xi^{0}, \eta\right)$ with respect to $\mu_{N}$.

We defer the proof of Lemma 3 to the appendix where it will be presented in a slightly more general form. Here we turn to

Proof of LemMa 4. As before we may assume that $\operatorname{rank} A(\eta)=\operatorname{rank} B(\eta)$ $=k$. In this proof we shall use the notations previously introduced in this section.

First we note that by (4.2), if $E$ is a $\mu_{k}$-measurable subset of $\pi_{k}(\zeta)$ (for some $\zeta$ in $R_{N-k}$ ) then

$$
\mathcal{H}_{k}\left(U_{0}(E)\right)=J_{k}(B(\eta)) \mu_{k}(E) .
$$

Let $c$ be a positive constant. We denote by $\mathcal{E}_{c}\left(x^{\prime}\right)$ the family of cubes in $R_{N}$ whose sides are parallel to the axes, such that

$$
\begin{array}{r}
E \in \mathcal{E}_{c}\left(x^{\prime}\right) \Rightarrow \exists r>0 \text { such that } E \subset\left\{x \in R_{n}:\left|x-x^{\prime}\right|<r\right\} \\
\quad \text { and } \mu_{N}(E) / r^{N}>c .
\end{array}
$$

Further we denote by $\mathscr{F}_{c}\left(\xi^{\prime}\right)$ the family of $\mathcal{T}_{k}$-measurable subsets of $\xi^{\prime}+M_{\eta}$ such that

$$
\begin{array}{r}
F \in \mathscr{F}_{c}\left(\xi^{\prime}\right) \Rightarrow \exists r>0 \text { such that } F \subset\left\{\xi \in \xi^{\prime}+M_{\eta}:\left|\xi-\xi^{\prime}\right|<r\right\} \\
\quad \text { and } \mathcal{K}_{k}(F) / r^{k}>c .
\end{array}
$$

Now let us consider the case $k=N$. If $E \in \mathcal{E}_{c}\left(x^{\prime}\right)$ then $E^{*}=U_{0}(E) \in$ $\mathscr{F}_{c^{\prime}}\left(\xi^{\prime}\right)$, where $c^{\prime}$ is a positive number depending only on $c$ and $\eta$. Indeed, if $E$ is contained in a ball of radius $r$ around $x^{\prime}$ then (in view of (4.3)) the set $E^{*}$ is contained in a ball of radius $|\eta| r$ around $\xi^{\prime}$. From this and (4.4) it follows that $E^{*} \in \mathscr{F}_{c^{\prime}}(\xi)$ if we choose, for instance, $c^{\prime}=J_{N}(B(\eta)) c|\eta|^{-N}$. Furthermore, by (4.2) and (4.4),

$$
\frac{1}{\mu_{N}(E)} \int_{E} f\left(x ; \xi^{0}, \eta\right) d \mu_{N}=\frac{1}{\mathcal{H}_{N}\left(E^{*}\right)} \int_{E^{*}} g(\xi, \eta) d \mathcal{C}_{N}(\xi)
$$


Since $\xi^{\prime} \in P(\eta)$, the right-hand side of (4.5) tends to $g\left(\xi^{\prime}, \eta\right)$ when $\mathcal{H}_{N}\left(E^{*}\right) \rightarrow$ 0 . This imples the assertion of the lemma when $k=N$.

Next, assume that $k<N$. If $E$ is a set in $R_{N}$ we denote by $E_{k}(\zeta)$ the set $E \cap \pi_{k}(\zeta), \zeta \in R_{N-k}$, and by $E_{N-k}$ the set

$$
\left\{\zeta \in R_{N-k}:\left(x_{1}, \ldots, x_{k}, \zeta_{1}, \ldots, \zeta_{N-k}\right) \in E \text { for some } x_{1}, \ldots, x_{k}\right\} \text {. }
$$

If $E \in \mathcal{E}_{c}\left(x^{\prime}\right)$ then $U_{0}\left(E_{k}(\zeta)\right) \in \mathscr{F}_{c^{\prime}}\left(\xi^{\prime}\right)$ for every $\zeta$ in $E_{N-k}$, where $c^{\prime}=$ $J_{k}(B(\eta)) c^{k / N}|\eta|^{-k}$. This can be verified in the same way as before. Now let $E$ be a cube in $\mathcal{E}_{c}\left(x^{\prime}\right)$ of side length $\delta$. Then, by (4.2) and (4.4),

$$
\begin{aligned}
\delta^{-N} \int_{E} f\left(x ; \xi^{0}, \eta\right) d \mu_{N} \\
\quad=\delta^{-N} \int_{E_{N-k}}\left(\int_{E_{k}(\zeta)} f\left(x ; \xi^{0}, \eta\right) d \mu_{k}\left(x_{1}, \ldots, x_{k}\right)\right) d \mu_{n-k}(\zeta) \\
=\delta^{-N+k} \int_{E_{N-k}}\left(\mathcal{H}_{k}(F(\zeta))^{-1} \int_{F(\zeta)} g(\xi, \eta) d \mathcal{H}_{k}\right) d \mu_{N-k}(\zeta)
\end{aligned}
$$

where $F(\zeta)=U_{0}\left(E_{k}(\zeta)\right)$. Since $\xi^{\prime} \in P(\eta), F(\zeta) \in \mathscr{F}_{c^{\prime}}\left(\xi^{\prime}\right)$ and $\mathcal{F}_{k}(F(\zeta))=$ const $\delta^{k}$ for every $\zeta$ in $E_{N-k}$ it follows that

$$
\mathcal{H}_{k}(F(\zeta))^{-1} \int_{F(\zeta)} g(\xi, \eta) d \mathcal{H}_{k} \rightarrow g\left(\xi^{\prime}, \eta\right)
$$

when $\delta \rightarrow 0$ uniformly with respect to $\zeta$ in $E_{N-k}$. Hence, by (4.6),

$$
\mu_{N}(E)^{-1} \int_{E} f\left(x ; \xi^{0}, \eta\right) d \mu_{N}(x) \rightarrow f\left(x^{\prime} ; \xi^{0}, \eta\right)
$$

when $E \in \mathcal{E}_{c}\left(x^{\prime}\right)$ and $\mu_{N}(E) \rightarrow 0$. This completes the proof of the lemma.

5. Proof of Theorem 2. First we note that it is sufficient to prove the theorem in the case where $g \geqslant 0$ and $r=1$. The result in the general case will follow immediately from this by considering the function $\tilde{g}=|g|^{1 / r}$. Secondly, we may assume that $\Omega$ is the unit cube $\left\{x \in R_{N}: 0<x_{i}<1\right\}$. Indeed, if $S_{g}$ maps $W_{1, p}(\Omega)$ into $L_{r}(\Omega)$ for some domain $\Omega$ in $R_{N}$, then

(i) $S_{g}$ maps $W_{1, p}\left(\Omega^{\prime}\right)^{m} \rightarrow L_{r}\left(\Omega^{\prime}\right)$ for any bounded domain $\Omega^{\prime}$ contained in $\Omega$, whose boundary is Lipschitz;

(ii) $S_{g}$ maps $W_{1, p}(\tilde{\Omega})^{m} \rightarrow L_{r}(\tilde{\Omega})$, where $\tilde{\Omega}$ is the image of $\Omega$ by a translation.

The first of these statements follows from the fact that any function in $W_{1, p}\left(\Omega^{\prime}\right)$ can be extended to a function in $W_{1, p}\left(R_{N}\right)$. The second statement is obvious.

Therefore in this section we shall consider Theorem 2 when $g \geqslant 0, r=1$ and $\Omega$ is the unit cube mentioned before.

Let $P(\eta)$ be defined as in $\S 4$ and let $M$ be a positive number. Set 


$$
\left\{\begin{array}{l}
Q=\left\{(\xi, \eta) \in R_{m} \times R_{m N}: \xi \in P(\eta)\right\} \\
Q_{M}=\{(\xi, \eta) \in Q:|\xi|<M\} .
\end{array}\right.
$$

Further denote by $G_{p}$ the real function on $R_{m} \times R_{m N}$ given by

$$
G_{p}(\xi, \eta)=1+|\xi|^{q}+|\eta|^{p}
$$

where $q=N p /(N-p)$ if $p<N$ and $q=0$ if $p>N$.

We shall prove the following assertions:

(5.3), If $1<p<N$ condition (2.3) holds everywhere in $Q$.

(5.3) $)_{2}$ If $p>N$ or $p \geqslant 1$ and $N=1$ condition (2.4) holds everywhere in $Q_{M}$.

Since $P(\eta)$ is a set of total measure in $R_{m}$ (Lemma 3 ) these assertions imply the statement of the theorem.

Suppose that assertion (5.3) (respectively (5.3) $)_{1}$ ) does not hold. Then there exists a sequence $\left\{\left(\xi^{n}, \eta^{n}\right)\right\}$ in $Q$ (resp. $\left.Q_{M}\right)$ such that

$$
g\left(\xi^{n}, \eta^{n}\right) / G_{p}\left(\xi^{n}, \eta^{n}\right)=\alpha_{n} \rightarrow \infty \quad \text { when } n \rightarrow \infty .
$$

We wish to show that this leads to a contradiction. To this purpose we shall consider several cases, in each of which, using (5.4), we shall construct functions $w_{1}, \ldots, w_{m}$ in $W_{1, p}(\Omega)$ such that $S_{g}\left(w_{1}, \ldots, w_{m}\right)$ is not in $L_{1}(\Omega)$.

Case I. Suppose that $1 \leqslant p<N$ and that there exists a positive number $\beta$ such that

$$
\beta<\left|\xi^{n}\right|^{q} /\left(1+\left|\eta^{n}\right|^{p}\right), \quad \text { where } q=N p /(N-p) .
$$

Let $\theta_{n}=1 / \alpha_{n}$. By considering a subsequence if necessary, we may assume that

Set

$$
\sum_{n=1}^{\infty} \theta_{n}^{1 / N} \leqslant \frac{1}{2} \beta^{1 / N} \quad \text { and } \quad \sum_{n=1}^{\infty} \theta_{n}^{(N-p) / N}<\infty
$$

$$
\delta_{n}^{N}=\theta_{n}\left|\xi^{n}\right|^{-q}, \quad n=1,2, \ldots
$$

Note that, in view of (5.5) and (5.6), $\Sigma_{1}^{\infty} \delta_{n} \leqslant \frac{1}{2}$.

By assumption $\xi^{n} \in P\left(\eta^{n}\right)$. Therefore, by Lemma $4, x=0$ is a Lebesgue point of the function $f\left(\cdot ; \xi^{n}, \eta^{n}\right)$ defined in (4.1). Hence, for every $c>0$ there exists a positive $\rho_{n}$ such that

$$
\mu_{N}(B)^{-1} \int_{B} f\left(\cdot ; \xi^{n}, \eta^{n}\right) d \mu_{N} \geqslant g\left(\xi^{n}, \eta^{n}\right) / 2,
$$

for every cube $B$ in $R_{N}$ such that $\mu_{N}(B) \leqslant \rho_{n}^{N}$ and the distance between $B$ and the origin is not larger than $c \mu_{N}(B)^{1 / N}$. In the sequel $\rho_{n}$ will correspond to $c=\sqrt{N} a_{N}^{-m / N}$, where $a_{N}$ is the constant appearing in (3.1) and (3.2).

Let $\sigma_{n}$ be the largest number of the form $\delta_{n} / 2 j, j=1,2, \ldots$, such that $\sigma_{n} \leqslant \rho_{n}$. Let $\left\{v_{i, n}\right\}_{n=1}^{m}$ be a set of functions in $E_{n}^{*}=\left\{x:\left|x_{i}\right| \leqslant \sigma_{n} / 2, i=\right.$ $1, \ldots, N\}$, constructed as in Lemma 2 with $\sigma=\sigma_{n}$ and $z^{i}=\left(\eta_{i, 1}^{n}, \ldots, \eta_{i, N}^{n}\right)$, 
$i=1, \ldots, m$. The cube $F$ mentioned in Lemma 2 will be denoted here by $F_{n}^{*}$. By (3.3) we have

$$
v_{i, n}(x)=\sum_{s=1}^{N} \eta_{i, s}^{n} x_{s}+b_{i, n}, \quad x \in F_{n}^{*},
$$

and

$$
\left|b_{i, n}\right|<2 \sqrt{N} \sigma_{n}\left|\left(\eta_{i, 1}, \ldots, \eta_{i, N}\right)\right|
$$

Let

$$
\left\{\begin{array}{l}
\gamma_{i, n}=\xi_{i}^{n}-b_{i, n}, \quad i=1, \ldots, m, \\
\tilde{v}_{i, n}(x)=v_{i, n}(x)+\gamma_{i, n} \quad \forall x \in E_{n}^{*}, i=1, \ldots, m .
\end{array}\right.
$$

Then,

$$
\left\{\begin{array}{l}
\tilde{v}_{i, n}(x)=\sum_{s=1}^{N} \eta_{i, s}^{n} x_{s}+\xi_{i}^{n} \quad \forall x \in F_{n}^{*}, i=1, \ldots, m, \\
\tilde{v}_{i, n}(x)=\gamma_{i, n} \quad \forall x \in \partial E_{n}^{*}, \text { and } \\
\left|\gamma_{i, n}\right|<\left|\xi_{i}^{n}\right|+2 \sqrt{N} \sigma_{n}\left|\left(\eta_{i, 1}^{n}, \ldots, \eta_{i, N}^{n}\right)\right| .
\end{array}\right.
$$

Next let $E_{n}=\left\{x:\left|x_{i}\right|<\delta_{n} / 2, i=1, \ldots, N\right\}$ and let $\left\{E_{n, v}\right\}_{\nu=1}^{k}$ be a family of cubes whose interiors are disjoint such that each cube $E_{n, y}$ is a translate of $E_{n}^{*}$ and $\cup_{v=1}^{k} E_{n, v}=E_{n}$. (These conditions uniquely determine the family $\left\{E_{n, \nu}\right\}$.) Denote by $x^{\nu}$ the center of the cube $E_{n, v}$. Then we define functions $u_{i, n}, i=1, \ldots, m$, in $E_{n}$ as follows

$$
u_{i, n}(x)=\tilde{v}_{i, n}\left(x-x^{\nu}\right), \quad \forall x \in E_{n, \nu}, \nu=1, \ldots, k \text {. }
$$

Let

$$
F_{n}=\bigcup_{\nu=1}^{k}\left(x^{\nu}+F_{n}^{*}\right) .
$$

Then by (5.8), (5.10), (5.11) and (3.2) we have

$$
\begin{aligned}
\int_{F_{n}} S_{g}\left(u_{1, n}, \ldots, u_{m, n}\right) d \mu_{N} & =k \int_{F_{n}^{*}} S_{g}\left(\tilde{v}_{1, n}, \ldots, \tilde{v}_{m, n}\right) d \mu_{N} \\
& >k \mu_{N}\left(F_{n}^{*}\right) g\left(\xi^{n}, \eta^{n}\right) / 2 \\
& >k \mu_{N}\left(E_{n}^{*}\right) a_{N}^{m} g\left(\xi^{n}, \eta^{n}\right) / 2=b_{1} \delta_{n}^{N} g\left(\xi^{n}, \eta^{n}\right)
\end{aligned}
$$

where $b_{1}=a_{N}^{m} / 2$. (Note that the distance between $F_{n}^{*}$ and the origin is not larger than $\sqrt{N}\left(\mu_{N}\left(F_{n}^{*}\right) / a_{N}^{m}\right)^{1 / N}$.)

Finally, set $D_{n}=\left\{x:\left|x_{i}\right|<\delta_{n}, i=1, \ldots, N\right\}$ and

$$
D_{n, \nu}=\left\{x: \delta_{n} / 2<\left|x_{\nu}\right|<\delta_{n},\left|x_{\nu}\right| \geqslant\left|x_{k}\right|, k=1, \ldots, N\right\}, \quad \nu=1, \ldots, N \text {. }
$$

Thus, $D_{n}=\left(\cup_{\nu=1}^{N} D_{n, v}\right) \cup E_{n}$. In $D_{n}$ we define $m$ functions $w_{1, n}, \ldots, w_{m, n}$ as follows: 


$$
\left\{\begin{array}{l}
w_{i, n}(x)=u_{i, n}(x) \quad \forall x \in E_{n}, \\
w_{i, n}(x)=2\left(\gamma_{i, n} / \delta_{n}\right) \cdot\left(\delta_{n}-\left|x_{v}\right|\right) \quad \forall x \in D_{n, v}, \nu=1, \ldots, N .
\end{array}\right.
$$

The functions $w_{1, n}, \ldots, w_{m, n}$ are continuous, piecewise linear in $D_{n}$, they vanish on $\partial D_{n}$ and their gradients satisfy the inequalities

$$
\left\{\begin{array}{l}
\left|\nabla w_{i, n}\right|<\left|\eta^{n}\right| \quad \text { in } E_{n} ; \\
\left|\nabla w_{i, n}\right|<2\left|\gamma_{i, n}\right| \delta_{n}^{-1}<2 \sqrt{N}\left(\left|\xi^{n}\right| \delta_{n}^{-1}+\left|\eta^{n}\right|\right) \quad \text { in } D_{n} \backslash E_{n}, i=1, \ldots, m .
\end{array}\right.
$$

For the second inequality we used (5.10).

Let $y^{n}$ be the point $\left(\tau_{n}, \frac{1}{2}, \ldots, \frac{1}{2}\right) \in R_{N}$, where $\tau_{n}=2 \sum_{1}^{n-1} \delta_{k}+\delta_{n}$, $n=1,2, \ldots$, and set $\tilde{D}_{n}=y^{n}+D_{n}$. Clearly, the interiors of the cubes $\tilde{D}_{n}$ are disjoint and (in view of the fact that $2 \Sigma_{1}^{\infty} \delta_{n}<1$ ) $\tilde{D}_{n} \subset \bar{\Omega}, n=1,2, \ldots$ Now, we define $m$ functions $w_{1}, \ldots, w_{m}$ in $\bar{\Omega}$ as follows:

$$
\begin{cases}w_{i}(x)=w_{i, n}\left(x-y^{n}\right) & \text { in } \tilde{D}_{n}, n=1,2, \ldots ; \\ w_{i}(x)=0 & \text { in } \bar{\Omega} \backslash \bigcup_{1}^{\infty} \tilde{D}_{n} .\end{cases}
$$

We claim that

$$
w_{i} \in W_{1, p}(\Omega), \quad i=1, \ldots, m
$$

and

$$
\int_{\Omega} S_{g}\left(w_{1}, \ldots, w_{m}\right) d \mu_{N}=\infty
$$

By construction the functions $w_{1}, \ldots, w_{m}$ are continuous in $\bar{\Omega}$, except at the point $\left(2 \Sigma_{1}^{\infty} \delta_{n}, \frac{1}{2}, \ldots, \frac{1}{2}\right)$, and vanish on $\partial \Omega$. Furthermore they are Lipschitz and, in fact, piecewise linear in every subset of $\bar{\Omega}$ which is bounded away from the point mentioned above. Therefore, in order to prove $(5.16)_{1}$ it is sufficient to show that $\nabla w_{i} \in L_{p}(\Omega)^{N}, i=1, \ldots, m$. By (5.14) and (5.15) we have

$$
\begin{aligned}
\int_{\tilde{D}_{n}}\left|\nabla w_{i}\right|^{p} d \mu_{N}=\int_{D_{n} \backslash E_{n}}\left|\nabla w_{i, n}\right|^{p} d \mu_{N}+\int_{E_{n}}\left|\nabla w_{i, n}\right|^{p} d \mu_{N} \\
\quad<\left(2^{N}-1\right) \delta_{n}^{N}(2 \sqrt{N})^{p}\left(\left|\xi^{n}\right| \delta_{n}^{-1}+\left|\eta^{n}\right|\right)^{p}+\delta_{n}^{N}\left|\eta^{n}\right|^{p} \\
\quad<\operatorname{const}\left(\delta_{n}^{N-p}\left|\xi^{n}\right|^{p}+\delta_{n}^{N}\left|\eta^{n}\right|^{p}\right), \quad i=1, \ldots, m ; n=1,2, \ldots
\end{aligned}
$$

By (5.5) and (5.7),

$$
\delta_{n}^{N}\left|\eta^{n}\right|^{p}<\delta_{n}^{N}\left|\xi^{n}\right|^{q} / \beta=\theta_{n} / \beta \quad \text { and } \quad \delta_{n}^{N-p}\left|\xi^{n}\right|^{p}=\theta_{n}^{(N-p) / N}
$$


Therefore, by (5.6) and (5.17),

$$
\int_{\Omega}\left|\nabla w_{i}\right|^{p} d \mu_{N} \leqslant \operatorname{const}\left(\sum_{n=1}^{\infty} \theta_{n}^{(N-p) / N}+\beta^{-1} \sum_{1}^{\infty} \theta_{n}\right)<\infty \quad(i=1, \ldots, m) .
$$

This proves $(5.16)_{1}$.

Now, by (5.15), (5.13) and (5.12) we have

$$
\begin{aligned}
\int_{\tilde{D}_{n}} S_{g}\left(w_{1}, \ldots, w_{m}\right) d \mu_{N}=\int_{D_{n}} S_{g}\left(w_{1, n}, \ldots, w_{m, n}\right) d \mu_{N} \\
\geqslant \int_{F_{n}} S_{g}\left(u_{1, n}, \ldots, u_{m, n}\right) d \mu_{N} \geqslant b_{1} g\left(\xi^{n}, \eta^{n}\right) \delta_{n}^{N} .
\end{aligned}
$$

Hence, using (5.4) and (5.7), we obtain

$$
\begin{array}{r}
\int_{\Omega} S_{g}\left(w_{1}, \ldots, w_{m}\right) d \mu_{N} \geqslant b_{1} \sum_{n=1}^{\infty} \delta_{n}^{N} g\left(\xi^{n}, \eta^{n}\right)=b_{1} \sum_{1}^{\infty} \delta_{n}^{N} \alpha_{n} G_{p}\left(\xi^{n}, \eta^{n}\right) \\
=b_{1} \sum_{1}^{\infty} \delta_{n}^{N} \theta_{n}^{-1}\left(1+\left|\xi^{n}\right|^{q}+\left|\eta^{n}\right|^{p}\right) \geqslant b_{1} \sum_{1}^{\infty} \delta_{n}^{N}\left|\xi^{n}\right|^{q} \theta_{n}^{-1}=+\infty .
\end{array}
$$

This proves $(5.16)_{2}$. Therefore we have proved that (5.4) and (5.5) lead to a contradiction.

Case II. Suppose that $1 \leqslant p<N$ and that

$$
\left|\xi^{n}\right|^{q} /\left(1+\left|\eta^{n}\right|^{p}\right) \leqslant \gamma \text { and } \beta^{1 / p}<\left|\eta^{n}\right|
$$

for some positive numbers $\beta, \gamma$. Let $\theta_{n}=1 / \alpha_{n}$. By considering a subsequence if necessary, we may assume that (5.6) holds. Set

$$
\delta_{n}^{N}=\theta_{n}\left|\eta^{n}\right|^{-p}, \quad n=1,2, \ldots
$$

In view of (5.19) and (5.6) we have $\Sigma_{1}^{\infty} \delta_{n} \leqslant \frac{1}{2}$.

Let $w_{1}, \ldots, w_{m}$ be defined exactly as in Case I. As before we shall show that (5.16) holds.

By (5.19) and (5.20)

$$
\begin{aligned}
\delta_{n}^{N-p}\left|\xi^{n}\right|^{p} & =\left(\delta_{n}^{N}\left|\xi^{n}\right|^{q}\right)^{(N-p) / N} \leqslant \operatorname{const}\left(\delta_{n}^{N}\left(1+\left|\eta^{n}\right|^{p}\right)\right)^{(N-p) / N} \\
& =\operatorname{const}\left(\delta_{n}^{N}+\theta_{n}\right)^{(N-p) / N}<\operatorname{const} \theta_{n}^{(N-p) / N}, \quad n=1,2, \ldots
\end{aligned}
$$

From this inequality together with (5.17) and (5.20) we obtain

$$
\int_{\tilde{D}_{n}}\left|\nabla w_{i}\right|^{p} d \mu_{N} \leqslant \operatorname{const}\left(\theta_{n}^{(N-p) / N}+\theta_{n}\right) \quad(i=1, \ldots, m ; n=1,2, \ldots) \text {. }
$$

Hence, by (5.6)

$$
\int_{\Omega}\left|\nabla w_{i}\right|^{p} d \mu_{N} \leqslant \operatorname{const}\left(\sum_{1}^{\infty} \theta_{n}^{(N-p) / N}+\sum_{1}^{\infty} \theta_{n}\right)<\infty, \quad i=1, \ldots, m .
$$

This implies $(5.16)_{1}$. 
Next, by (5.18) and (5.20),

$$
\begin{aligned}
\int_{\Omega} S_{g}\left(w_{1}, \ldots, w_{m}\right) d \mu_{N} & \geqslant b_{1} \sum_{n=1}^{\infty} \delta_{n}^{N} g\left(\xi^{n}, \eta^{n}\right)=b_{1} \sum_{n=1}^{\infty} \delta_{n}^{N} \alpha_{n} G_{p}\left(\xi^{n}, \eta^{n}\right) \\
& \geqslant b_{1} \sum_{n=1}^{\infty} \delta_{n}^{N} \alpha_{n}\left|\eta^{n}\right|^{p}=b_{1} \sum_{1}^{\infty} \alpha_{n} \theta_{n}=\infty .
\end{aligned}
$$

Thus we have shown that (5.16) holds. Therefore (5.4) and (5.19) lead to a contradiction.

Case III. Suppose that $1 \leqslant p<\infty$ and that

$$
\left|\xi^{n}\right|+\left|\eta^{n}\right| \leqslant C, \quad n=1,2, \ldots
$$

for some constant $C$.

By considering a subsequence if necessary, we may assume that

$$
\xi^{n} \rightarrow \xi^{0} \text { and } \sum_{1}^{\infty}\left|\xi^{n}-\xi^{0}\right|<\infty
$$

and also that

$$
\sum_{1}^{\infty} \alpha_{n}^{-1}<\frac{1}{2}
$$

Let $\rho_{n}, \sigma_{n}$ and $\tilde{v}_{i, n}$ be as in Case I with $\delta_{n}=\alpha_{n}^{-1}$. Set

$$
\begin{aligned}
& J_{n}=\left\{x: 0 \leqslant x_{1} \leqslant \delta_{n}, 0<x_{i}<1, i=2, \ldots, N\right\}, \\
& \tilde{J}_{n}=\left\{x: 0 \leqslant x_{1} \leqslant \delta_{n}, 0 \leqslant x_{i} \leqslant \kappa_{n}, i=2, \ldots, N\right\},
\end{aligned}
$$

where $\kappa_{n}$ is the largest integral multiple of $\sigma_{n}$ such that $\kappa_{n}<1$. Note that, since $\sigma_{n}<\frac{1}{2}$, we have $\kappa_{n} \geqslant \frac{1}{2}$.

Let $\left\{J_{n, \nu}\right\}_{\nu=1}^{s}$ be a family of cubes whose interiors are disjoint such that the union of the cubes equals $\tilde{J}_{n}$ and each cube $J_{n, \nu}$ is a translate of $E_{n}^{*}$. Denote by $x^{\nu}$ the center of the cube $J_{n, \nu}$. Now we define in $J_{n}$ functions $u_{1, n}, \ldots, u_{m, n}$ as follows:

$$
\begin{cases}u_{i, n}(x)=\tilde{v}_{i, n}\left(x-x^{\nu}\right), & \forall x \in J_{n, \nu}, \nu=1, \ldots, s ; \\ u_{i, n}(x)=\gamma_{i, n}, & \forall x \in J_{n} \backslash \tilde{J}_{n},\end{cases}
$$

with $\gamma_{i, n}$ as in (5.9). Let $F_{n}=\cup_{v=1}^{s}\left(x^{\nu}+F_{n}^{*}\right)$ where $F_{n}^{*}$ is as in Case I. Then, by (5.24), (5.10), (5.8), (5.4) and (3.2) we have

$$
\begin{aligned}
\int S_{g}\left(u_{1, n}, \ldots, u_{m, n}\right) d \mu_{N}=s \int_{F_{n}^{*}} S_{g}\left(\tilde{v}_{1, n}, \ldots, \tilde{v}_{m, n}\right) d \mu_{N} \\
\geqslant s \mu_{N}\left(E_{n}^{*}\right) a_{N}^{m} g\left(\xi^{n}, \eta^{n}\right) / 2=\delta_{n} \kappa_{n} a_{N}^{m} g\left(\xi^{n}, \eta^{n}\right) / 2 \\
\geqslant\left(a_{N}^{m} / 4\right) \alpha_{n}^{-1} g\left(\xi^{n}, \eta^{n}\right)>a_{N}^{m} / 4 .
\end{aligned}
$$


Next let $\beta=2 \Sigma_{1}^{\infty}\left|\Gamma_{n+1}-\Gamma_{n}\right|$, where $\Gamma_{n}=\left(\gamma_{1, n}, \ldots, \gamma_{m, n}\right)$. Note that by (5.21) $-(5.23)$ and (5.9) $\beta$ is finite. Indeed,

$$
\left|\Gamma_{n}-\xi^{n}\right|<2 \sqrt{N} \sigma_{n}\left|\eta^{n}\right|<\sqrt{\dot{N}} \delta_{n}\left|\eta^{n}\right|
$$

and hence,

$$
\left|\Gamma_{n+1}-\Gamma_{n}\right|<\left|\xi^{n+1}-\xi^{n}\right|+\sqrt{N}\left(\delta_{n+1}\left|\eta^{n+1}\right|+\delta_{n}\left|\eta^{n}\right|\right)<\left|\xi^{n+1}-\xi^{n}\right|+\sqrt{N} C .
$$

Let $J_{n}^{*}=\left\{x: 0<x_{1}<\delta_{n}^{*}, 0<x_{i}<1, i=2, \ldots, N\right\}$, where

$$
\delta_{n}^{*}= \begin{cases}\left|\Gamma_{n+1}-\Gamma_{n}\right| \beta^{-1} & \text { if } \beta \neq 0 \\ 0 & \text { if } \beta=0\end{cases}
$$

In $J_{n}^{*}$ we define functions $u_{i, n}^{*}(i=1, \ldots, m)$ by

$$
\begin{cases}u_{i, n}^{*}(x)=\left(\left(\gamma_{i, n+1}-\gamma_{i, n}\right) / \delta_{n}^{*}\right) x_{1}+\gamma_{i, n} & \text { if } \delta_{n}^{*}>0, \\ u_{i, n}^{*}(x)=\gamma_{i, n} & \text { if } \delta_{n}^{*}=0, \forall x \in J_{n}^{*} .\end{cases}
$$

We are now ready to define functions $w_{1}, \ldots, w_{m}$ in $\Omega$ which will be shown to satisfy (5.16). Let $e^{1}=(1,0, \ldots, 0)$ and set $I_{n}=\beta_{n} e^{1}+J_{n}, I_{n}^{*}=\beta_{n}^{*} e^{1}+$ $J_{n}^{*}$ where

$$
\begin{gathered}
\beta_{1}=0, \quad \beta_{n}=\sum_{1}^{n-1}\left(\delta_{k}+\delta_{k}^{*}\right), \quad n=2,3, \ldots ; \\
\beta_{n}^{*}=\beta_{n+1}-\delta_{n}^{*}, \quad n=1,2 \ldots
\end{gathered}
$$

Let

$$
\begin{cases}w_{i}(x)=u_{i, n}\left(x-\beta_{n} e^{1}\right), & \forall x \in I_{n}, \quad n=1,2 \ldots, \\ w_{i}(x)=u_{i, n}^{*}\left(x-\beta_{n}^{*} e^{1}\right), & \forall x \in I_{n}^{*}, \quad n=1,2 \ldots, \\ w_{i}(x)=\xi^{0}, & \forall x \in \bar{\Omega} \backslash \cup_{i}^{\infty}\left(I_{n}+I_{n}^{*}\right), \quad i=1, \ldots, m,\end{cases}
$$

with $\xi^{0}$ as in (5.22).

The function $w_{i}$ is bounded and continuous in $\bar{\Omega}$ and it is piecewise linear in every subdomain bounded away from the hyperplane $x_{1}=\Sigma_{1}^{\infty}\left(\delta_{n}+\delta_{n}^{*}\right)$. Furthermore, by (5.21), (5.24), (5.27) and (5.28),

$$
\left|\nabla w_{i}\right|<C \text { in } I_{n} \text { and }\left|\nabla w_{i}\right|<\beta \text { in } I_{n}^{*} \text {. }
$$

Hence $w_{i} \in W_{1, \infty}(\Omega)$. 
It remains to show that $w_{1}, \ldots, w_{m}$ satisfy $(5.16)_{2}$. By (5.28) and (5.25),

$$
\begin{aligned}
\int_{\Omega} S_{g} & \left(w_{1}, \ldots, w_{m}\right) d \mu_{N} \geqslant \sum_{n=1}^{\infty} \int_{I_{n}} S_{g}\left(w_{1}, \ldots, w_{m}\right) d \mu_{N} \\
& =\sum_{n=1}^{\infty} \int_{J_{n}} S_{g}\left(u_{1, m}, \ldots, u_{m, n}\right) d \mu_{N} \geqslant \sum_{n=1}^{\infty} \int_{F_{n}} S_{g}\left(u_{1, n}, \ldots, u_{m, n}\right) d \mu_{N}=\infty .
\end{aligned}
$$

Thus (5.4) and (5.21) lead to a contradiction.

Case IV. Suppose that $1<p<\infty$ and that

$$
\left|\xi^{n}\right| \leqslant C_{0}, \quad C_{1}<\left|\eta^{n}\right|^{p}
$$

for some positive $C_{0}, C_{1}$. By considering a subsequence if necessary we may assume $\Sigma_{1}^{\infty} \alpha_{n}^{-1} \leqslant C_{1} / 2$ and that (5.22) holds. Set

$$
\delta_{n}=\left(\alpha_{n}\left|\eta^{n}\right|^{p}\right)^{-1}
$$

Note that $\Sigma_{1}^{\infty} \delta_{n} \leqslant \Sigma_{1}^{\infty}\left(\alpha_{n} C_{1}\right)^{-1}<\frac{1}{2}$. Let $\beta$ be as in Case III. By (5.22), (5.26), (5.29) and (5.30), $\beta$ is finite. Indeed,

$$
\begin{aligned}
\left|\Gamma_{n+1}-\Gamma_{n}\right| & \leqslant\left|\xi^{n+1}-\xi^{n}\right|+\sqrt{N}\left(\delta_{n+1}\left|\eta^{n+1}\right|+\delta_{n}\left|\eta^{n}\right|\right) \\
& <\left|\xi^{n+1}-\xi^{n}\right|+\sqrt{N} C_{1}^{(p-1) / p}\left(\alpha_{n+1}^{-1}+\alpha_{n}^{-1}\right) .
\end{aligned}
$$

Let $\delta_{n}^{*}$ be as in Case III. Define $u_{i, n}, u_{i, n}^{*}$ and $w_{i}$ as in the previous case.

The function $w_{i}$ is piecewise linear in $I_{n}$ and

$$
w_{i}=\gamma_{i, n} \text { on } \partial I_{n}, \quad\left|\nabla w_{i}\right|<\left|\eta^{n}\right| \text { in } I_{n} \quad(n=1,2, \ldots) .
$$

Thus $\left|w_{i}-\gamma_{i, n}\right| \leqslant\left|\eta^{n}\right| \delta_{n}<C_{1}^{(p-1) / p} \alpha_{n}^{-1}$ everywhere in $I_{n}$. Furthermore, by (5.26), $\left|\xi_{i}^{n}-\gamma_{i, n}\right| \leqslant \sqrt{N} \delta_{n}\left|\eta^{n}\right|<$ const $\alpha_{n}^{-1}(n=1,2, \ldots)$. Finally, in $I_{n}^{*}$ $(n=1,2, \ldots), w_{i}$ is linear and its range lies between $\gamma_{i, n}$ and $\gamma_{i, n+1}$. Therefore (in view of (5.22)) $w_{i}$ is bounded and continuous in $\Omega$ and $w_{i}$ is piecewise linear in every subdomain bounded away from the hyperplane $x_{1}=$ $\Sigma_{1}^{\infty}\left(\delta_{n}+\delta_{n}^{*}\right)$. In addition, by (5.24), (5.27), (5.28) and (5.30)

$$
\begin{gathered}
\int_{\Omega}\left|\nabla w_{i}\right|^{p} d \mu_{N}=\sum_{1}^{\infty}\left(\int_{I_{n}}\left|\nabla w_{i}\right|^{p} d \mu_{N}+\int_{I_{n}^{*}}\left|\nabla w_{i}\right|^{p} d \mu_{N}\right) \\
\leqslant \sum_{1}^{\infty}\left[\left|\eta^{n}\right|^{p} \delta_{n}+\left(\frac{\left|\Gamma_{n+1}-\Gamma_{n}\right|}{\delta_{n}^{*}}\right)^{p} \delta_{n}^{*}\right] \\
=\sum_{1}^{\infty} \alpha_{n}^{-1}+\beta^{p-1} \sum_{1}^{\infty}\left|\Gamma_{n+1}-\Gamma_{n}\right|<\infty .
\end{gathered}
$$

Thus $(5.16)_{1}$ holds. 
By (5.4), (5.25), (5.28) and (5.30),

$$
\begin{aligned}
\int_{\Omega} S_{g}\left(w_{1}, \ldots, w_{m}\right) d \mu_{N} \geqslant \sum_{1}^{\infty} \int_{F_{n}} S_{g}\left(u_{1, n}, \ldots, u_{m, n}\right) d \mu_{N} \\
\geqslant\left(\frac{a_{N}^{m}}{4}\right) \sum_{1}^{\infty} \delta_{n} g\left(\xi^{n}, \eta^{n}\right) \geqslant\left(\frac{a_{N}^{m}}{4}\right) \sum_{1}^{\infty}\left(\alpha_{n}\left|\eta^{n}\right|^{p}\right)^{-1} \alpha_{n}\left(1+\left|\eta^{n}\right|^{p}\right)=\infty
\end{aligned}
$$

Thus (5.16) 2 holds. This shows that (5.4) and (5.29) lead to a contradiction.

We claim that the contradictions obtained in Cases I-IV prove (5.3), and (5.3) $)_{2}$. Clearly, if one of the assumptions (5.5), (5.19), (5.21) or (5.29) holds for a subsequence of $\left\{\left(\xi^{n}, \eta^{n}\right)\right\}$ then (5.4) is impossible. Thus, Case III shows that if $\left\{\left(\xi^{n}, \eta^{n}\right)\right\}$ contains a bounded subsequence then we cannot have (5.4). Now suppose that $\left\{\xi^{n}\right\}$ is bounded. Then we must have $\left|\eta^{n}\right| \rightarrow \infty$ and Case IV show that (5.4) is impossible. This already proves (5.3)

Next, if $1 \leqslant p<N$, Case I shows that (5.4) cannot hold unless

$$
\lim \left|\xi^{n}\right|^{q} /\left(1+\left|\eta^{n}\right|^{p}\right)=0 \text {. }
$$

If (5.31) holds and if $\left\{\eta^{n}\right\}$ contains a subsequence that is bounded away from zero then Case II shows that (5.4) is impossible. On the other hand if (5.31) holds and $\eta^{n} \rightarrow 0$ then the sequence $\left\{\left(\xi^{n}, \eta^{n}\right)\right\}$ is bounded and this again contradicts (5.4). This proves (5.3) 1 and completes the proof of the theorem.

6. Proof of Theorem 1; the necessity of the conditions. We start with the case $m=1$. Thus $h: R \rightarrow R$ is a Borel function such that $T_{h}$ maps $W_{1, p}(\Omega)$ into $W_{1, r}(\Omega)$. Without loss of generality we may assume that $\Omega$ is the unit cube $\left\{x: 0<x_{i}<1, i=1, \ldots, N\right\}$.

First we claim that $h$ is l.a.c. (i.e. locally absolutely continuous) in $R$. Let $u(x)=x_{1}-c$ where $c$ is an arbitrary constant. Since $u \in W_{1, p}(\Omega)$, we have $h \circ u \in W_{1, r}(\Omega)$. This implies that there exists an 1.a.c. function $h_{0}: R \rightarrow R$ such that $h=h_{0}$ a.e. in $R$. Suppose that there exists a point $\xi_{0}$ such that $h\left(\xi_{0}\right) \neq h_{0}\left(\xi_{0}\right)$. Consider the function $u$ given by

$$
u(x)= \begin{cases}\left(x_{1}-\frac{1}{2}\right)+\xi_{0}, & \frac{1}{2} \leqslant x_{1}<1, \\ \xi_{0}, & 0<x_{1}<\frac{1}{2}, \forall x \in \Omega .\end{cases}
$$

Let $v=h \circ u$. Thus $v(x)=h\left(x_{1}-\frac{1}{2}+\xi_{0}\right)=h_{0}\left(x_{1}-\frac{1}{2}+\xi_{0}\right)$ a.e. in the set $\left\{x \in \Omega: \frac{1}{2}<x_{1}\right\}$ and $v(x)=h\left(\xi_{0}\right) \neq h_{0}\left(\xi_{0}\right)$ in $\left\{x \in \Omega: x_{1} \leqslant \frac{1}{2}\right\}$. Therefore $\partial v / \partial x_{1}$ (in the distribution sense) is not a function. On the other hand, by assumption, $v \in W_{1, r}(\Omega)$ because $u \in W_{1, p}(\Omega)$. This contradiction shows that $h$ is identical with $h_{0}$.

Let $u \in W_{1,1}^{\text {loc }}(\Omega)$. Since $h$ is 1.a.c., $h \circ u \in W_{1,1}^{\text {loc }}(\Omega)$ if and only if $\left(h^{*} \circ u\right) \nabla u$ $\in L_{1}^{\text {loc }}(\Omega)^{N}$, where $h^{*}: R \rightarrow R$ is any Borel function which is equivalent to $h^{\prime}$. Moreover $\nabla(h \circ u)=\left(h^{*} \circ u\right) \nabla u$ a.e. in $\Omega$. For a proof of this result see [4]. 
Therefore, if $g: R \times R_{N} \rightarrow R$ is defined by

$$
g(\xi, \eta)=\left|h^{*}(\xi) \eta\right|, \quad(\xi, \eta) \in R \times R_{N},
$$

then $S_{g}$ maps $W_{1, p}(\Omega)$ into $L_{r}(\Omega)$. Hence, by Theorem 2 , if $1<r<p<N$, we have

$$
g(\xi, \eta) \leqslant \operatorname{const}\left(1+|\xi|^{q / r}+|\eta|^{p / r}\right), \quad q=N p /(N-p)
$$

for every $\eta$ in $R_{N}$ and every $\xi$ in $R \backslash C_{\eta}$ where $C_{\eta}$ is a null subset of $R$ which may depend on $\eta$. In the present case, $g$ is continuous with respect to $\eta$. Therefore the above statement implies that there exists a null subset of $R$, say $A$, such that (6.2) holds for every $(\xi, \eta)$ in $(R \backslash A) \times R_{N}$. In fact if $D$ is a countable dense subset of $R_{N}$ we could set $A=\cup_{\eta \in D} C_{\eta}$.

Similarly, if $p>N$ (or $p \geqslant 1$ and $N=1$ ) and $M>0$, we have

$$
g(\xi, \eta) \leqslant c(M)\left(1+|\eta|^{p / r}\right),
$$

for every $(\xi, \eta)$ in $(R \backslash A) \times R_{N}$ such that $|\xi| \leqslant M$, with $A$ (as before) a null subset of $R$.

From (6.1), (6.3) we deduce that $h^{*}$ is locally essentially bounded and hence that $h$ is locally Lipschitz in $R$.

If $p<N$, (6.2) implies that

$$
\left|h^{*}(\xi)\right|<\text { const } \inf _{0<\gamma<\infty} H(|\xi|, \gamma), \quad \xi \in R \backslash A,
$$

where

$$
H(\beta, \gamma)=\left(1+\beta^{q / r}+\gamma^{p / r}\right) / \gamma \quad(0<\beta, \gamma)
$$

A simple computation show that (6.4) and (6.5) imply that

$$
\left|h^{*}(\xi)\right|<\operatorname{const}\left(1+|\xi|^{\nu}\right) \quad \forall \xi \in R \backslash A \text {, where } \nu=N(p-r) /(r(N-p)) \text {. }
$$

This completes the proof in the case $m=1$.

Next we consider the case $m>1$. Thus $h: R_{m} \rightarrow R$ is a Borel function such that $T_{h}$ maps $W_{1, p}(\Omega)^{m}$ into $W_{1, r}(\Omega)$. Again we may assume that $\Omega$ is the unit cube.

Let $f_{i}: R \rightarrow R, i=1, \ldots, m$, be uniformly Lipschitz functions. Let $\tilde{h}$ : $R \rightarrow R$ be the function given by

$$
\tilde{h}(t)=h\left(f_{1}(t), \ldots, f_{m}(t)\right), \quad \forall t \in R .
$$

If $u \in W_{1, p}(\Omega)$ then $f_{i} \circ u \in W_{1, p}(\Omega), i=1, \ldots, m$. Therefore $T_{\tilde{h}}$ maps $W_{1, p}(\Omega)$ into $W_{1, r}(\Omega)$. Hence, by the first part of the proof, $\tilde{h}$ is locally Lipschitz in $R$. This implies in particular that $h$ is locally Lipschitz on every line parallel to one of the axes. 
Now we claim that $h$ is continuous and moreover

$$
\underset{\xi \rightarrow \xi^{0}}{\lim \sup }\left|h(\xi)-h\left(\xi^{0}\right)\right| /\left|\xi-\xi^{0}\right|=M\left(\xi^{0}\right)<\infty, \quad \forall \xi^{0} \in R_{m} .
$$

Suppose that (6.8) fails at some point $\xi^{0}$. Then there exists a sequence $\left\{\xi^{n}\right\}$ in $R_{m}$ such that $\xi^{n} \rightarrow \xi^{0}$ and

$$
\left|h\left(\xi^{n}\right)-h\left(\xi^{0}\right)\right|>n\left|\xi^{n}-\xi^{0}\right|, \quad n=1,2, \ldots
$$

By considering a subsequence if necessary, we may assume that

$$
\left|\xi^{n}-\xi^{0}\right|>2\left|\xi^{n+1}-\xi^{0}\right|, \quad n=1,2, \ldots
$$

Let

$\alpha=\sum_{k=1}^{\infty}\left|\xi^{k+1}-\xi^{k}\right|, \quad \alpha_{n+1}=\sum_{k=1}^{n}\left|\xi^{k+1}-\xi^{k}\right| \quad(n=1,2, \ldots)$ and $\alpha_{1}=0$.

In view of (6.10) $\alpha$ is finite. We define a curve $\xi=\xi(t), 0<t<\alpha$, as follows.

$$
\begin{aligned}
& \xi(t)=\xi^{n}+\left(\left(t-\alpha_{n}\right) /\left(\alpha_{n+1}-\alpha_{n}\right)\right)\left(\xi^{n+1}-\xi^{n}\right), \\
& \xi(\alpha)=\xi^{0} \\
& t \in\left[\alpha_{n}, \alpha_{n+1}\right], n=1,2,
\end{aligned}
$$

Clearly, $\left|\xi(t)-\xi\left(t^{\prime}\right)\right| \leqslant\left|t-t^{\prime}\right|$ for every $t, t^{\prime}$ in $[0, \alpha]$. Therefore the function $\tilde{h}$ given by $\tilde{h}(t)=h(\xi(t))$ is Lipschitz in [0, $\alpha$ ]. Thus, using (6.10),

$$
\left|h\left(\xi^{n}\right)-h\left(\xi^{0}\right)\right|=\left|\tilde{h}\left(\alpha_{n}\right)-\tilde{h}(\alpha)\right|<\mathrm{const}\left|\xi^{n}-\xi^{0}\right|, \quad n=1,2, \ldots
$$

But this contradicts (6.9).

By Stepanov's theorem [1, p. 218], (6.8) implies that $h$ possesses a differential a.e. in $R_{m}$. Furthermore, since $h$ is continuous, the set $B$ where its differential is defined is a Borel set and the partial derivatives $\partial h / \partial \xi_{i}$, $i=1, \ldots, m$, are Borel functions on $B[1$, p. 211].

Let $g: R_{m} \times R_{m N} \rightarrow[0, \infty)$ be the function given by

$$
g(\xi, \eta)= \begin{cases}\left|\sum_{i=1}^{m} \frac{\partial h}{\partial \xi_{i}}(\xi)\left(\eta_{i, 1}, \ldots, \eta_{i, N}\right)\right|, & \text { if } \xi \in B, \\ 0, & \text { if } \xi \in R_{m} \backslash B .\end{cases}
$$

(As usual we write $\eta=\left(\eta_{1,1}, \ldots, \eta_{1, N}, \ldots, \eta_{m, 1}, \ldots, \eta_{m, N}\right)$.) Clearly $g$ is a Borel function. We claim that

$$
\mathbf{u}=\left(u_{1}, \ldots, u_{m}\right) \in W_{1, p}(\Omega)^{m} \Rightarrow S_{g}(\mathbf{u}) \in L_{r}(\Omega) .
$$

Since $\mathbf{u}$ belongs to $W_{1, p}(\Omega)^{m}$, it is equivalent to a function $\mathbf{u}^{*}$ such that, for a.e. line $\tau$ parallel to one of the axes in $R_{N},\left.u^{*}\right|_{\tau \cap \Omega}$ is 1.a.c. Furthermore $\partial u^{*} / \partial x_{j}$ (the partial derivative in the classical sense) exists a.e. in $\Omega$ and is equivalent to the corresponding distribution derivative of $u, j=1, \ldots, m$ 
(see [7, §3.1]). Without loss of generality we may assume that $\mathbf{u}$ is identical with $\mathbf{u}^{*}$. Then the function $h \circ \mathbf{u}$, which by assumption belongs to $W_{1, r}(\Omega)$, is continuous in $\tau \cap \Omega$ for a.e. line $\tau$ parallel to one of the axes. (Recall that $h$ is continuous.) It follows from this that $h \circ \mathbf{u}$ is l.a.c. in $\tau \cap \Omega$ for a.e. $\tau$ as above. Now, if $x^{0}$ is a point in $\Omega$ such that $\mathrm{u}\left(x^{0}\right) \in B$ and such that $\partial \mathrm{u} / \partial x_{j}$ exists at $x^{0}$ then $\partial(h \circ u) / \partial x_{j}$ exists at $x^{0}$ and

$$
\left(\frac{\partial(h \circ \mathbf{u})}{\partial x_{j}}\right)\left(x^{0}\right)=\sum_{i=1}^{m}\left(\frac{\partial h}{\partial \xi_{i}}\right)\left(\mathbf{u}\left(x^{0}\right)\right)\left(\frac{\partial u_{i}}{\partial x_{j}}\right)\left(x^{0}\right) .
$$

Hence, by (6.11), $S_{g}(u)=|\nabla(h \circ u)|$ a.e. in $B^{*}=\{x \in \Omega: u(x) \in B\}$ and $S_{g}(\mathbf{u})=0$ in $\Omega \backslash B^{*}$. Since $h \circ \mathbf{u} \in W_{1, r}(\Omega)$, this implies that $S_{g}(\mathbf{u}) \in L_{r}(\Omega)$.

Suppose that $1 \leqslant r \leqslant p<N$. By Theorem 2, (6.12) implies that $g$ satisfies inequality (6.2) for every $\eta$ in $R_{N}$ and every $\xi$ in $R_{m} \backslash C_{\eta}$, where $C_{\eta}$ is a null set. From this we deduce, by the same argument as in the case $m=1$, that the partial derivatives of $h$ satisfy the inequality

$$
\left|\frac{\partial h}{\partial \xi_{i}}(\xi)\right|<\operatorname{const}\left(1+|\xi|^{n}\right) \quad \text { a.e. in } R_{m}, i=1, \ldots, m,
$$

with $\nu$ as in (2.2).

Similarly when $p>N$ (or $p \geqslant 1$ if $N=1$ ) we deduce that for every $M>0$ there exists a constant $\kappa_{M}$ such that

$$
\left|\frac{\partial h}{\partial \xi_{i}}(\xi)\right| \leqslant \kappa_{M} \quad \text { a.e. in the sphere }|\xi| \leqslant M, i=1, \ldots, m .
$$

By (6.13) and (6.14) the partial derivatives $\partial h / \partial \xi_{i}(i=1, \ldots, m)$ are locally essentially bounded in $R_{m}$. This, together with the fact that $h$ is continuous in $R_{m}$ and locally Lipschitz on every line parallel to one of the axes, implies that $h$ is locally Lipschitz in $R_{m}$. This completes the proof of the necessity part of Theorem 1 .

7. Proof of Theorem 1; the sufficiency of the conditions.. The sufficiency of the conditions of Theorem 1 could be derived from [5, Theorems 2.1 and 3.3] in which a much more general situation was considered. However in order to apply the results of [5] directly to our case we would have to assume that the inequality in (2.1) holds at every point where $\partial h / \partial \xi_{i}$ exists. An examination of the arguments in [5] shows that, in the present case, this stronger condition can be replaced by (2.1). However, for the convenience of the reader we shall present here a simple, independent proof of the sufficiency part of Theorem 1.

First some general remarks. Let $A(\Omega)$ denote the space of real measurable functions in $\Omega$ such that $v \in A(\Omega)$ iff for almost every line $\tau$ parallel to one of the axes $\left.v\right|_{\tau \cap \Omega}$ is 1.a.c. (i.e. locally absolutely continuous). A function $u$ 
belongs to $W_{1, p}(\Omega)$ if and only if $u$ is equivalent to a function $u^{*}$ belonging to $A(\Omega)$ such that $u^{*}$ and $\partial u^{*} / \partial x_{i}(i=1, \ldots, N)$ belong to $L_{p}(\Omega)$. Here $\partial u^{*} / \partial x_{i}$ denotes the classical partial derivative of $u^{*}$. (It is known that $\partial u^{*} / \partial x_{i}$ is defined a.e. in $\Omega$ and is measurable.) Furthermore $\partial u^{*} / \partial x_{i}$ is equivalent to the corresponding distribution derivative of $u$. (See [7], [2].)

If $h: R_{m} \rightarrow R$ is locally Lipschitz and $\mathbf{u}=\left(u_{1}, \ldots, u_{m}\right) \in A(\Omega)^{m}$ then, clearly $h \circ \mathrm{u} \in A(\Omega)$.

We turn now to the proof of the sufficiency part of Theorem 1 and we start with the case where $p>N$ or $N=1$ and $p \geqslant 1$. In this case every function $u \in W_{1, p}(\Omega)$ is equivalent to a function in $C(\Omega)$ and

$$
\|u\|_{L_{\infty}(\Omega)} \leqslant c_{1}\|u\|_{W_{1, p}(\Omega)}
$$

where $c_{1}$ is a constant which depends on $\Omega$.

Let $\mathbf{u} \in W_{1, p}(\Omega)^{m}$. Without loss of generality we may assume that $\mathbf{u} \in$ $C(\Omega)^{m}$. This implies that $\mathbf{u} \in A(\Omega)^{m}$ and hence $h \circ \mathbf{u} \in A(\Omega)$. Let $\|u\|_{W_{1, p}(\Omega)}$ $\leqslant M$ and let $b_{M}$ be the Lipschitz constant of $h$ in the sphere $|\xi|<c_{1} M$. Then, by (7.1)

$$
|h \circ \mathbf{u}(x)-h(\mathbf{0})| \leqslant b_{M}|\mathbf{u}(x)|
$$

and therefore

$$
\|h \circ \mathbf{u}\|_{L_{p}(\Omega)} \leqslant|h(0)| \mu_{N}(\Omega)^{1 / p}+b_{M}\|\mathbf{u}\|_{L_{p}(\Omega)}
$$

Let $\tau$ be a line parallel to the $x_{i}$-axis such that $\left.u\right|_{\tau \cap \Omega}$ is l.a.c. and let $x, x^{\prime}$ be two points in $\tau \cap \Omega$. Since

$$
\left|h \circ \mathbf{u}(x)-h \circ \mathbf{u}\left(x^{\prime}\right)\right| \leqslant b_{M}\left|\mathbf{u}(x)-\mathbf{u}\left(x^{\prime}\right)\right|,
$$

dividing by $\left|x-x^{\prime}\right|$ and letting $x^{\prime}$ tend to $x$ we obtain

$$
\left|\partial(h \circ \mathbf{u}) / \partial x_{i}\right| \leqslant b_{M}\left|\partial \mathbf{u} / \partial x_{i}\right| \quad \mu_{1} \text {-a.e. on } \tau \cap \Omega \text {. }
$$

In view of the fact that $h \circ \mathbf{u} \in A(\Omega),(7.2)$ and (7.3) imply that $h \circ \mathbf{u} \in$ $W_{1, p}(\Omega)$ and

$$
\|h \circ \mathbf{u}\| W_{1, \rho}(\Omega)<|h(0)| \mu_{N}(\Omega)^{1 / p}+b_{M}\|\mathbf{u}\| W_{1, \rho}(\Omega)^{m} .
$$

Next we consider the case where $1 \leqslant r \leqslant p \leqslant N$. Let $Z_{k}(k=1, \ldots, m)$ be the family of lines $\{\tau\}$ in $R_{m}$, parallel to the $\xi_{k}$ axis, such that (2.1) holds $\mu_{1}$-a.e. on $\tau$. The projection of $Z_{k}$ on the hyperplane $\xi_{k}=0$ is a set of total measure (with respect to $\mu_{m-1}$ ) in this hyperplane.

Given two points $\xi, \xi^{\prime}$ in $R_{m}$ we shall say that they are connected through $Z_{1}, \ldots, Z_{m}$ if there exists a continuous piecewise linear curve $C$ connecting $\xi$ with $\xi^{\prime}$, which consists of $j$ linear segments $C_{1}, \ldots, C_{j}$ such that $C_{i}$ is a segment of a line belonging to $Z_{k_{i}}(i=1, \ldots, j)$ and $k_{1}, \ldots, k_{j}$ are distinct. Suppose that $\xi, \xi^{\prime}$ have this property. Then, by (2.1), 


$$
\left|h(\xi)-h\left(\xi^{\prime}\right)\right|<\sum_{i=1}^{j} \int_{C_{i}}\left|\frac{\partial h}{\partial \xi_{k_{i}}}\right| d \mu_{1}<m a_{0}\left(1+|\xi|^{\nu}+\left|\xi^{\prime}\right|^{\nu}\right)\left|\xi-\xi^{\prime}\right|
$$

Now, if $\xi, \xi^{\prime}$ are two arbitrary points in $R_{m}$ there exist sequences of points in $R_{m}$, say $\left\{\xi^{n}\right\}$ and $\left\{\xi^{\prime \prime}\right\}$, such that $\xi^{n} \rightarrow \xi, \xi^{\prime n} \rightarrow \xi^{\prime}$ and each pair $\xi^{n}, \xi^{\prime n}$ is connected through $Z_{1}, \ldots, Z_{m}$. Therefore

$$
\left|h\left(\xi^{n}\right)-h\left(\xi^{\prime n}\right)\right|<m a_{0}\left(1+\left|\xi^{n}\right|^{\nu}+\left|\xi^{\prime n}\right|^{\nu}\right)\left|\xi^{n}-\xi^{\prime n}\right|
$$

for every $n$ and by continuity

$$
\left|h(\xi)-h\left(\xi^{\prime}\right)\right|<m a_{0}\left(1+|\xi|^{\nu}+\left|\xi^{\prime}\right|^{\nu}\right)\left|\xi-\xi^{\prime}\right| \text {. }
$$

Now let $\mathbf{u} \in W_{1, p}(\Omega)^{m}$. Without loss of generality we may assume that $\mathbf{u}$ belongs also to $A(\Omega)^{m}$ and so $h \circ \mathrm{u} \in A(\Omega)$. By (7.5),

$$
|h \circ \mathrm{u}(x)|<|h(0)|+m a_{0}\left(1+|\mathrm{u}(x)|^{\nu}\right)|\mathrm{u}(x)| \text {. }
$$

Hence, by Sobolev's imbedding theorem

$$
\begin{aligned}
\|h \circ \mathbf{u}\|_{L_{r}(\Omega)} & <|h(0)| \mu_{N}(\Omega)^{1 / r}+m a_{0}\left(\|\mathbf{u}\|_{L_{r}(\Omega)}+\|\mathbf{u}\|_{L_{q}(\Omega)}^{\nu}\|\mathbf{u}\|_{L_{p}(\Omega)}\right) \\
& <|h(0)| \mu_{N}(\Omega)^{1, r}+\text { const }\|\mathbf{u}\|_{W_{1}(\Omega)}^{p+1}
\end{aligned}
$$

where the constant depends on $\Omega$ and $q=N p /(N-p)$.

Let $\tau$ be a line parallel to the $x_{i}$-axis such that $\left.u\right|_{\tau \cap \Omega}$ is 1.a.c. and let $x, x^{\prime} \in \tau \cap \Omega$. Then by (7.5),

$$
\left|h \circ \mathbf{u}(x)-h \circ \mathbf{u}\left(x^{\prime}\right)\right|<m a_{0}\left(1+|\mathbf{u}(x)|^{\nu}+\left|\mathbf{u}\left(x^{\prime}\right)\right|^{\nu}\right)\left|\mathbf{u}(x)-\mathbf{u}\left(x^{\prime}\right)\right| \text {. }
$$

Hence

$$
\left|\partial(h \circ \mathbf{u}) / \partial x_{i}\right|<m a_{0}\left(1+2|\mathbf{u}(x)|^{\nu}\right)\left|\partial \mathrm{u} / \partial x_{i}\right|
$$

$\mu_{1}$-a.e. in $\tau \cap \Omega$. This inequality implies

$$
\begin{aligned}
\left\|\partial(h \circ \mathrm{u}) / \partial x_{i}\right\|_{L_{r}(\Omega)} & <2 m a_{0}\left(\left\|\partial \mathrm{u} / \partial x_{i}\right\|_{L_{r}(\Omega)}+\|\mathrm{u}\|_{L_{q}(\Omega)}\left\|\partial \mathrm{u} / \partial x_{i}\right\|_{L_{P}(\Omega)}\right) \\
& <\text { const }\|\mathbf{u}\| W_{W_{\rho}(\Omega)}^{p+1}
\end{aligned}
$$

where the constant depends on $\Omega$. In view of the fact that $h \circ u \in A(\Omega),(7.6)$ and (7.7) imply that $h \circ u \in W_{1, r}(\Omega)$ and

$$
\|h \circ \mathbf{u}\|_{W_{1, r}(\Omega)}<\operatorname{const}\left(1+\|\mathrm{u}\|_{W_{1,}(\omega)}^{\nu+1}\right) \text {. }
$$

This completes the proof of Theorem 1.

8. The case of unbounded domains. Let $\Omega$ be an unbounded domain in $R_{N}$ satisfying the cone condition. Then we have the following result, parallel to Theorems 1 and 2. 
TheOREM 3. Let $h: R_{m} \rightarrow R$ be a Borel function and let $r, p$ be two numbers such that $1<r \leqslant p<N$. Then $T_{h}$ maps $W_{1, p}(\Omega)^{m}$ into $W_{1, r}(\Omega)$ if and only if the following conditions hold.

(i) $h(0)=0$ and $h$ is locally Lipschitz in $R_{m}$.

(ii) The first order partial derivatives of $h$ satisfy the inequality

$$
\left|\frac{\partial h}{\partial \xi_{i}}(\xi)\right| \leqslant a_{0}\left(|\xi|^{\nu}+|\xi|^{\nu^{\prime}}\right) \quad \text { a.e. in } R_{m}, i=1, \ldots, m \text {, }
$$

where $a_{0}$ is a constant, and $\nu=N(p-r) /(r(N-p))$ and $\nu^{\prime}=(p-r) / r$.

If $p>N$ (or $N=1$ and $p>1$ ) and $r=p$ then $T_{h}$ maps $W_{1, p}(\Omega)^{m}$ into $W_{1, r}(\Omega)$ if and only if (i) holds.

In either case, if $T_{h}$ maps $W_{1, p}(\Omega)^{m}$ into $W_{1, r}(\Omega)$ then the mapping is bounded and the following inequalities hold:

$$
\begin{aligned}
& \left\|T_{h} \mathrm{u}\right\|_{W_{1, \rho}(\Omega)}<b_{0} \sum_{i=1}^{m}\left(\left\|u_{i}\right\|_{W_{1 p}(\Omega)}^{p+1}+\left\|u_{i}\right\|_{W_{1 / p}(\Omega)}^{\nu^{\prime}+1}\right), \quad \text { if } p<N \\
& \left\|T_{h} \mathrm{u}\right\|_{W_{1, p}(\Omega)}<b(M) \sum_{i=1}^{m}\left\|u_{i}\right\|_{W_{1, p}(\Omega)}, \quad \text { if } p>N(\text { or } p>1 \text { when } N=1) \\
& \quad \text { and } \sum_{i=1}^{m}\left\|u_{i}\right\|_{W_{1, p}(\Omega)}<M .
\end{aligned}
$$

The constants $b_{0}$ and $b(M)$ depend on $m, a_{0}$ and $\Omega$.

THEOREM 4. Let $g, p, r$ be as in Theorem 2. Suppose that $S_{g}$ maps $W_{1, p}(\Omega)^{m}$ into $L_{r}(\Omega)$ (where $\Omega$ is unbounded). Then $g$ satisfies the conditions described in Theorem 2 with (2.3) and (2.4) replaced by the first and second of the inequalities below:

$$
\begin{aligned}
& |g(\xi, \eta)| \leqslant C_{0}\left(|\xi|^{q / r}+|\xi|^{p / r}+|\eta|^{p / r}\right), \\
& |g(\xi, \eta)| \leqslant C(M)\left(|\xi|^{p / r}+|\eta|^{p / r}\right) .
\end{aligned}
$$

In addition we have $\mathrm{g}(0,0)=0$.

We shall start with the proof of Theorem 4, in which we use the result of Theorem 2. Then, using Theorems 1 and 4, we shall prove Theorem 3.

Proof OF THEOREM 4. Without loss of generality we shall assume that $g>0$ and $r=1$. As in the proof of Theorem 2 we shall show that:

(8.5) 1 If $1 \leqslant p<N$ then (8.3) holds everywhere in $Q$.

(8.5) $)_{2}$ If $p>N$ or $p \geqslant 1$ and $N=1$ then (8.4) holds everywhere in $Q_{M}$.

If $\Omega^{\prime}$ is a cube contained in $\Omega$ then $S_{g}$ maps $W_{1, p}\left(\Omega^{\prime}\right)^{m}$ into $L_{1}\left(\Omega^{\prime}\right)$. Therefore $g$ satisfies inequality (2.3) in $Q$ when $p<N$ and inequality (2.4) in $Q_{M}$ when $p>N$ or $p>1$ and $N=1$. But inequality (8.3) (resp. (8.4)) is equivalent to inequality (2.3) (resp. (2.4)) in every domain of the form $\{(\xi, \eta):|\xi|+|\eta|>a$ $>0\}$. Therefore, if either $(8.5)_{1}$ or $(8.5)_{2}$ fails then there must exist a sequence 
$\left\{\left(\xi^{n}, \eta^{n}\right)\right\}$ in $Q$ such that

$$
\left|\xi^{n}\right|+\left|\eta^{n}\right| \rightarrow 0
$$

and

$$
g\left(\xi^{n}, \eta^{n}\right) /\left(\left|\xi^{n}\right|^{p}+\left|\eta^{n}\right|^{p}\right)=\alpha_{n} \rightarrow \infty \quad \text { as } n \rightarrow \infty .
$$

We shall show that (8.6) and (8.7) lead to a contradiction. But first some general remarks.

By considering a subsequence of $\left\{\left(\xi^{n}, \eta^{n}\right)\right\}$ if necessary, we may assume that

$$
\sum_{1}^{\infty} \alpha_{n}^{-1}<\infty \quad \text { and } \quad \sum_{1}^{\infty}\left(\left|\xi^{n}\right|^{p}+\left|\eta^{n}\right|^{p}\right)<\infty
$$

Since $\Omega$ is unbounded and satisfies the cone condition, it follows that there exists an infinite family $\mathcal{Q}$ of disjoint closed cubes of equal volume such that each cube is contained in $\Omega$ and its sides are parallel to the axes. We shall denote by $2 \lambda$ the side length of these cubes.

Now we shall consider two cases in each of which we shall show that (8.6) and (8.7) lead to a contradiction to the assumptions of the theorem. The conclusion of the theorem will follow directly from these results.

Case (a). Suppose that there exists a positive $\beta$ such that

$$
g\left(\xi^{n}, \eta^{n}\right)=\alpha_{n}\left(\left|\xi^{n}\right|^{p}+\left|\eta^{n}\right|^{p}\right)<\beta, \quad n=1,2, \ldots
$$

In view of $(8.7)$ it is clear that $g\left(\eta^{n}, \eta^{n}\right) \neq 0$ for sufficiently large $n$; we may assume that $g\left(\xi^{n}, \eta^{n}\right) \neq 0$ for all $n$. Let

$$
K_{n}=\left[\beta g\left(\xi^{n}, \eta^{n}\right)^{-1}\right]+1
$$

where (for $b>0$ ) $[b]$ denotes the largest integer that is not larger than $b$. Note that, in view of (8.9),

$$
K_{n} \leqslant 2 \beta g\left(\xi^{n}, \eta^{n}\right)^{-1} \text {. }
$$

Let $\left\{\mathbb{Q}_{n}\right\}^{\infty}$ be a partition of $\mathbb{Q}$ such that $\mathbb{Q}_{n}$ consists of exactly $K_{n}$ cubes, $A_{n, j}\left(j=1, \ldots, K_{n}\right)$. Let $w_{i, n}$ be defined as in the proof of Theorem 2 (see (5.13)) with $D_{n}, E_{n}$ replaced by

$$
D=\left\{x:\left|x_{i}\right|<\lambda, i=1, \ldots, R\right\}, \quad E=\left\{x:\left|x_{i}\right|<\lambda / 2, i=1, \ldots, N\right\} .
$$

Then, by (5.9) $-(5.11),(5.13)$ and (5.14) we have

$$
\begin{cases}\left|w_{i, n}(x)\right|<\left|\gamma_{i, n}\right|+\lambda\left|\eta^{n}\right|<\operatorname{const}\left(\left|\xi^{n}\right|+\left|\eta^{n}\right|\right), & \forall x \in D, \\ \left|\nabla w_{i, n}(x)\right|<\left|\eta^{n}\right|, & \text { a.e. in } E, \\ \left|\nabla w_{i, n}(x)\right|<2\left|\gamma_{i, n}\right| \lambda^{-1}<\operatorname{const}\left(\left|\xi^{n}\right|+\left|\eta^{n}\right|\right), & \text { a.e. in } D \backslash E,\end{cases}
$$


$n=1,2, \ldots$ Now we define $m$ functions $w_{1}, \ldots, w_{m}$ in $\bar{\Omega}$ as follows:

$\begin{cases}w_{i}(x)=w_{i, n}\left(x-x^{n, j}\right), & \forall x \in A_{n, j}\left(n=1,2, \ldots ; j=1, \ldots, K_{n}\right), \\ w_{i}(x)=0, & x \in \bar{\Omega} \backslash \Omega_{0},\end{cases}$

where $x^{n j}$ is the center of the cube $A_{n, j}$ and $\Omega_{0}=\cup\{A: A \in \mathbb{Q}\}$.

Let $\tilde{D}_{n}=\cup_{j=1}^{K_{n}} A_{n j}$. Then by (8.9)-(8.13),

$$
\begin{aligned}
\int_{\tilde{D}_{n}}\left|w_{i}\right|^{p} d \mu_{N} & <\text { const } K_{n} \lambda^{N}\left(\left|\xi^{n}\right|+\left|\eta^{n}\right|\right)^{p}<\text { const } K_{n}\left(\left|\xi^{n}\right|^{p}+\left|\eta^{n}\right|^{p}\right) \\
& \leqslant \text { const } \alpha_{n}^{-1}, \quad n=1,2, \ldots
\end{aligned}
$$

and

$$
\int_{\tilde{D}_{n}}\left|\nabla w_{i}\right|^{p} d \mu_{N}<\text { const } K_{N} \lambda^{N}\left(\left|\xi^{n}\right|+\left|\eta^{n}\right|\right)^{p}<\text { const } \alpha_{n}^{-1}, \quad n=1,2, \ldots
$$

Therefore, by (8.8), $w_{i}$ and $\left|\nabla w_{i}\right|$ belong to $L_{p}(\Omega), i=1, \ldots, m$. In addition, $w_{i}$ is locally Lipschitz in $\Omega$; hence, $w_{i} \in W_{1, p}(\Omega), i=1, \ldots, m$.

On the other hand, by (8.10), (8.11), (8.13) and (5.12), (5.13) we have

$$
\begin{aligned}
\int_{\tilde{D}_{n}} S_{g}\left(w_{1}, \ldots, w_{m}\right) d \mu_{N}=K_{n} \int_{D} S_{g}\left(w_{1, n}, \ldots, w_{m, n}\right) d \mu_{N} \\
\quad>K_{n} \int_{E} S_{g}\left(u_{1, n}, \ldots, u_{m, n}\right) d \mu_{N} \geqslant K_{n} b_{1} \lambda^{N} g\left(\xi^{n}, \eta^{n}\right)>b_{1} \lambda^{N} \beta .
\end{aligned}
$$

Hence $S_{g}\left(w_{1}, \ldots, w_{m}\right) \notin L_{1}(\Omega)$ and we have reached a contradiction.

Case (b). Suppose that

$$
g\left(\xi^{n}, \eta^{n}\right)=\alpha_{n}\left(\left|\xi^{n}\right|^{p}+\left|\eta^{n}\right|^{p}\right) \rightarrow \infty \quad \text { as } n \rightarrow \infty .
$$

Then we may assume that

$$
g\left(\xi^{n}, \eta^{n}\right)^{-1}<\lambda^{N}, \quad n=1,2, \ldots
$$

Let

$$
\delta_{n}^{N}=g\left(\xi^{n}, \eta^{n}\right)^{-1}
$$

Note that $\delta_{n}<\lambda$.

Let $u_{i, n}, i=1, \ldots, m$, be $m$ functions defined in the cube $E_{n}=\{x$ : $\left.\left|x_{i}\right| \leqslant \delta_{n} / 2, i=1, \ldots, N\right\}$ as in the proof of Theorem 2 (see (5.11)). Set

$$
D_{n}=D=\left\{x:\left|x_{i}\right|<\lambda, i=1, \ldots, N\right\}
$$

and

$$
D_{n, \nu}=\left\{x: \delta_{n} / 2 \leqslant\left|x_{\nu}\right| \leqslant \lambda,\left|x_{\nu}\right| \geqslant\left|x_{k}\right|, k=1, \ldots, N\right\}, \quad \nu=1, \ldots, N .
$$

Note that $D=\left(\cup_{\nu=1}^{N} D_{n, v}\right) \cup E_{n}$. Now, for every $n$ we define functions $w_{i, n}$, $i=1, \ldots, m$, in $D$ as follows, 
$\left\{\begin{array}{l}w_{i, n}(x)=u_{i, n}(x), \quad \forall x \in E_{n}, \\ w_{i, n}(x)=\gamma_{i, n}\left(\lambda-\delta_{n} / 2\right)^{-1}\left(\lambda-\left|x_{\nu}\right|\right), \quad \forall x \in D_{n, v}, \nu=1, \ldots, N .\end{array}\right.$

Then, by (5.9)-(5.11),

$$
\begin{cases}\left|w_{i, n}\right|<\left|\gamma_{i, n}\right|+\delta_{n}\left|\eta^{n}\right|<\operatorname{const}\left(\left|\xi^{n}\right|+\left|\eta^{n}\right|\right), & \text { in } D, \\ \left|\nabla w_{i, n}\right|<\left|\eta^{n}\right|, & \text { in } E_{n} \\ \left|\nabla w_{i, n}\right|<\left|\gamma_{i, n}\right|\left(\lambda-\delta_{n} / 2\right)^{-1}<\operatorname{const}\left(\left|\xi^{n}\right|+\left|\eta^{n}\right|\right), & \text { in } D \backslash E_{n}\end{cases}
$$

Here we have used also the fact that $\delta_{n}<\lambda$.

Let us denote the cubes in $Q$ by $A_{n}, n=1,2, \ldots$, and let $x^{n}$ be the center of $A_{n}$. Then we define functions $w_{1}, \ldots, w_{m}$ in $\bar{\Omega}$ as follows,

$$
\begin{cases}w_{i}(x)=w_{i, n}\left(x-x^{n}\right), & \forall x \in A_{n}, n=1,2, \ldots \\ w_{i}(x)=0, & \forall x \in \bar{\Omega} \backslash \cup_{1}^{\infty} A_{n}, i=1, \ldots, m .\end{cases}
$$

By (8.18).

$$
\int_{A_{n}}\left|w_{i}\right|^{p} d \mu_{N}=\int_{D}\left|w_{i, n}\right|^{p} d \mu_{N}<\operatorname{const}\left(\left|\xi^{n}\right|+\left|\eta^{n}\right|\right)^{p} \lambda^{N}
$$

and

$$
\int_{A_{n}}\left|\nabla w_{i}\right|^{p} d \mu_{N}=\int_{D}\left|\nabla w_{i, n}\right|^{p} d \mu_{N}<\operatorname{const}\left(\left|\xi^{n}\right|+\left|\eta^{n}\right|\right)^{p} \lambda^{N}
$$

Hence, by (8.8), $w_{i}$ and $\left|\nabla w_{i}\right|$ belong to $L_{p}(\Omega)$ and so $w_{i} \in W_{1, p}(\Omega)$. On the other hand, by (8.19), (8.17), (8.16) and (5.12),

$$
\begin{aligned}
\int_{A_{n}} S_{g}\left(w_{1}, \ldots, w_{m}\right) d \mu_{N} & =\int_{D} S_{g}\left(w_{1, n}, \ldots, w_{m, n}\right) d \mu_{N} \\
& \geqslant \int_{E_{n}} S_{g}\left(u_{1, n}, \ldots, u_{m, n}\right) d \mu_{N} \\
& \geqslant b_{1} \delta_{n}^{N} g\left(\xi^{n}, \eta^{n}\right)=b_{1} .
\end{aligned}
$$

Thus $S_{g}\left(w_{1}, \ldots, w_{m}\right) \notin L_{1}(\Omega)$ and again we have reached a contradiction.

To complete the proof of the theorem we note that the sequence $\left\{\left(\xi^{n}, \eta^{n}\right)\right\}$ must contain a subsequence for which either (8.9) or (8.14) holds. Therefore (8.6) and (8.7) are incompatible with the assumption that $S_{g}$ maps $W_{1, p}(\Omega)^{m}$ into $L_{1}(\Omega)$.This establishes (8.5) and $(8.5)_{2}$ and hence (8.3) and (8.4).

Finally, since $\Omega$ is unbounded and satisfies the cone condition it has infinite measure. Therefore the fact that $g$ vanishes for $(\xi, \eta)=(0,0)$ is obvious; otherwise $S_{g}(0, \ldots, 0)$ would not belong to $L_{1}(\Omega)$.

Next we turn to 
Proof of Theorem 3. Suppose that $T_{h}$ maps $W_{1, p}(\Omega)^{m}$ into $W_{1, r}(\Omega)$. If $\Omega^{\prime}$ is a bounded subdomain of $\Omega$ whose boundary is Lipschitz, then $T_{h}$ maps $W_{1, p}\left(\Omega^{\prime}\right)^{m}$ into $W_{1, r}\left(\Omega^{\prime}\right)$. Therefore, by Theorem $2, h$ is locally Lipschitz in $R_{m}$. The fact that $h(0)=0$ is obvious. In order to prove the growth estimate in the case $p<N$ we apply Theorem 4 . Using this theorem one can show, by the same arguments that were used in $\$ 6$, that

$$
\left|\frac{\partial h}{\partial \xi_{i}}(\xi)\right| \leqslant \text { const } \inf _{0<\gamma<\infty} \tilde{H}(|\xi|, \gamma) \quad \text { a.e. in } R_{m}, i=1, \ldots, m,
$$

where

$$
\tilde{H}(\beta, \gamma)=\left(\beta^{p / r}+\beta^{q / r}+\gamma^{p / r}\right) / \gamma, \quad \beta, \gamma>0 .
$$

Hence (considering the cases $|\xi|<1$ and $|\xi|>1$ separately) one obtains (8.1) by a simple calculation.

The sufficiency of the conditions stated in the theorem and inequality (8.2) can be verified by the same arguments that were used in the proof of Theorem 1 (\$7).

9. Further remarks and open questions. In this section we shall discuss two additional problems related to the mappings $S_{g}$ and $T_{h}$, for which only partial results are available. For simplicity we shall consider only the case where $\Omega$ is a bounded domain in $R_{N}$ satisfying the cone condition.

The first problem is to obtain necessary and sufficient conditions in order that $S_{g}$ map $W_{1, p}(\Omega)^{m}$ into $L_{r}(\Omega)$. Theorem 2 provides necessary conditions. However, when $m>1$, these conditions are obviously not sufficient because of the exceptional sets where the inequalities (2.3) or (2.4) may fail. We describe below several results which may help to clarify the problem stated above. But first a definition.

Given a measurable set $B$ in $R_{m}$ we shall say that it is a null projection set if the projection of $B$ on each of the coordinate axes is a $\mu_{1}$-null set.

THEOREM 5. Let $g: R_{m} \times R_{m N} \rightarrow R$ be a Borel function and let $p, r$ be two numbers such that $1 \leqslant r \leqslant p<\infty$. Suppose that

$$
g(\xi, 0)=0 \quad \forall \xi \in R_{m} .
$$

Suppose that there exists a null projection set $B$ in $R_{m}$ such that if $p<N$ then (2.3) holds everywhere in $\left(R_{m} \backslash B\right) \times R_{m N}$ and if $p>N$ (or $N=1$ and $\left.p>1\right)$ then (2.4) holds everywhere in $\left(R_{m} \backslash B\right) \times R_{m N}$. Then $S_{g}$ maps $W_{1, p}(\Omega)^{m}$ into $L_{r}(\Omega)$.

Proof. Let $w=\left(w_{1}, \ldots, w_{m}\right) \in W_{1, p}(\Omega)^{m}$. Denote by $B^{(i)}$ the projection of $B$ on the $\xi_{i}$ axis $(i=1, \ldots, m)$. Since $B^{(i)}$ is null it follows that $\nabla w_{i}=0$ a.e. in $w_{i}^{-1}\left(B^{(i)}\right)$ (see [4]). Therefore, by $(9.1), S_{g}(w)=0$ a.e. in $w^{-1}(B)$. In view of this fact, applying Sobolev's imbedding theorem, (2.3) and (2.4) imply that $S_{g}(w) \in L_{r}(\Omega)$. 
Using Theorems 2 and 5 we obtain,

TheOREM 6. Suppose that $g: R_{m} \times R_{m N}$ is a Borel function satisfying (9.1) and suppose that there exists a constant $K_{0}$ such that

$$
\begin{aligned}
|g(\xi, \eta)|<K_{0} \limsup _{r \rightarrow 0} r^{-m} \int_{\left|\xi^{\prime}\right|<r}\left|g\left(\xi+\xi^{\prime}, \eta\right)\right| d \xi^{\prime}, & \\
& \forall(\xi, \eta) \in\left(R_{m} \backslash B\right) \times R_{m N},
\end{aligned}
$$

where $B$ is a null projection set.

Then the conditions stated in Theorem 2 are necessary and sufficient in order that $S_{g}$ should map $W_{1, p}(\Omega)^{m}$ into $L_{r}(\Omega)$.

Proof. We have to verify only the sufficiency of the conditions. In view of (9.2) it is clear that (2.3) or (2.4) hold everywhere in $\left(R_{m} \backslash B\right) \times R_{m N}$. Therefore by Theorem 5 these conditions are sufficient.

TheOREM 7. Suppose that $g: R \times R_{N} \rightarrow R$ is a Borel function satisfying (9.1), such that $g(\xi, \cdot)$ is continuous in $R_{N}$ for a.e. $\xi$ in $R$. Then the conditions stated in Theorem 2 are necessary and sufficient for $S_{g}$ to map $W_{1, p}(\Omega)$ into $L_{r}(\Omega)$.

Proof. Again we have to verify only the sufficiency of the conditions stated in Theorem 2. The continuity of $g$ with respect to $\eta$ implies that (2.3) or (2.4) holds everywhere in $(R \backslash B) \times R_{N}$, where $B$ is a null subset of $R$. Therefore, by Theorem 5 these conditions are sufficient.

We conjecture that the result of Theorem 7 holds without the assumption that $g$ is continuous with respect to $\eta$. At present we have established this conjecture in the case $N=1$. However the proof is rather lengthy and we shall not present it here.

The second problem that we wish to mention is that of the continuity of the mapping $T_{h}$. It is known that if $h \in C^{1}\left(R_{m}\right)$ and $h$ satisfies the conditions of Theorem 1 then $T_{h}: W_{1, p}(\Omega)^{m} \rightarrow W_{1, p}(\Omega)$ is continuous. However, if $m=1$, the assumption that $h \in C^{1}\left(R_{m}\right)$ is not necessary. Indeed, in [6] we showed that, if $m=1, T_{h}$ is a continuous mapping of $W_{1, p}(\Omega)$ into $W_{1, r}(\Omega)$ whenever $h$ satisfies the conditions of Theorem 1 . The question of whether this result is valid when $m>1$ is still unresolved.

10. An additional result concerning the mapping $T_{h}$. In this section we consider superposition mappings of the type (1.1) where $h$ depends also on the space variable $x$. As before we shall assume that $\Omega$ is a domain in $R_{N}$ satisfying the cone condition.

Let $h$ be a real function on $\Omega \times R_{m}$ and set

$$
\left(T_{h} \mathbf{u}\right)(x)=h(x, \mathbf{u}(x)), \quad \forall x \in \Omega,
$$

for every $R_{m}$-valued function $\mathrm{u}$ in $\Omega$. We shall present below an extension of 
the sufficiency part of Theorem 1 for mappings of this type. The sufficient conditions are quite general and they may provide a good indication of the type of conditions that are also necessary in order that $T_{h}$ should map $W_{1, p}(\Omega)^{m}$ into $W_{1, r}(\Omega)$. However the problem of obtaining a full extension of Theorem 1 to mappings of the type (10.1) is still unresolved.

The following notations will be used: $D_{x_{i}} v$ will denote the distribution derivative with respect to $x_{i}$ of a function $v$ in $L_{1}^{\text {loc }}(\Omega) ; \partial V / \partial \xi_{i}$ will denote the (classical) partial derivative with respect to $\xi_{i}$ of a function $V$ in $R_{m}$.

First we bring,

LEMMA 5. Let $\Omega$ be bounded and let $p, r$ be two numbers such that either $1<r<p<\infty$ and $p \neq N$ or $r=p=1$. Suppose that $h$ is $a$ real function on $\boldsymbol{\Omega} \times \boldsymbol{R}_{m}$ satisfying the conditions:

(i) $h(\cdot, \xi) \in W_{1, r}(\Omega)$ for every $\xi$ in $R_{m}$.

(ii) $h(x, \cdot)$ is locally Lipschitz in $R_{m}$ for a.e. $x$ in $\Omega$.

In addition, if $p<N$ assume that:

(iii) There exists a countable set $E$, dense in $R_{m}$, such that

$\left|D_{x_{i}} h(x, \xi)\right| \leqslant f(x)+b|\xi|^{q / r}, \quad \forall \xi \in E$, a.e. $x$ in $\Omega, i=1, \ldots, N$,

where $f \in L_{r}(\Omega), b$ is a constant and $q=N p /(N-p)$.

(iv) For a.e. $x$ in $\Omega$,

$$
\left|\left(\partial h / \partial \xi_{i}\right)(x, \xi)\right|<f^{*}(x)+b^{*}|\xi|^{\prime}, \quad \text { a.e. in } R_{m}, i=1, \ldots, m,(10.3)
$$

where $f^{*} \in L_{s}(\Omega)$ with $s=p r /(p-r), b^{*}$ is a constant and $\nu=N(p-$ $r) /(r(N-p))$. (The exceptional null set in $R_{m}$ where $(10.3)$ does not hold may vary with $x$.)

If $p>N$ or $p>1$ and $N=1$, assume that the following conditions hold for every $a>0$. (Here $E$ and $s$ will be as before.)

(iii)' There exists $f_{a} \in L_{r}(\Omega)$ such that

$$
\left|D_{x_{i}} h(x, \xi)\right| \leqslant f_{a}(x), \quad i=1, \ldots, N,
$$

for every $\xi$ in $E$ such that $|\xi| \leqslant a$ and a.e. $x$ in $\Omega$.

(iv)' Fore a.e. $x$ in $\Omega$,

$$
\left|\left(\partial h / \partial \xi_{i}\right)(x, \xi)\right| \leqslant f_{a}^{*}(x), \quad \text { a.e. in }\left\{\xi \in R_{m}:|\xi|<a\right\}, i=1, \ldots, m \text {, }
$$

where $f_{a}^{*} \in L_{s}(\Omega)$.

Under these assumptions $T_{h}$ maps $W_{1, p}(\Omega)^{m}$ into $W_{1, r}(\Omega)$ and the mapping is bounded. Furthermore, the following pointwise estimates hold for $\mathbf{u} \in W_{1, p}(\Omega)^{m}$. If $p<N$ then

$$
\begin{aligned}
& \left|T_{h} \mathrm{u}(x)\right|<\mathrm{const}\left(|h(x, 0)|+f^{*}(x)|\mathrm{u}(x)|+|\mathrm{u}(x)|^{p+1}\right) \\
& \left|D_{x_{i}}\left(T_{h} \mathrm{u}\right)(x)\right|<\operatorname{const}\left(|f(x)|+|\mathrm{u}(x)|^{q / r}+\left(f^{*}(x)+|\mathrm{u}(x)|^{\nu}\right)\left|D_{x_{i}} \mathrm{u}\right|\right)
\end{aligned}
$$


a.e. in $\Omega, i=1, \ldots, N$. If $p>N$ or $p \geqslant 1$ and $N=1$, and ess $\sup _{\Omega}|u(x)|<a$ then

$$
\begin{aligned}
\left|T_{h} \mathbf{u}(x)\right| & \leqslant \operatorname{const}\left(|h(x, 0)|+f_{a}^{*}(x)|\mathbf{u}(x)|\right) ; \\
\left|D_{x_{i}}\left(T_{h} \mathbf{u}\right)(x)\right| & \leqslant \operatorname{const}\left(f_{a}(x)+f_{a}^{*}(x)\left|D_{x_{i}} \mathbf{u}\right|\right)
\end{aligned}
$$

a.e. in $\Omega, i=1, \ldots, N$. The constants depend only on $m$, and $b^{*}$.

REMARK 1. In the case $r=1, r<p$ the lemma remains valid if we make the following additional assumptions:

$h \in C\left(\Omega \times R_{m}\right) ; h(x, \cdot)$ is locally Lipschitz for every $x$ in $\Omega$;

(10.3) and (10.5) hold for every $x$ in $\Omega$ and $f^{*}, f_{a}^{*}$ are finite everywhere in $\Omega$.

REMARK 2. If $\Omega$ is an arbitrary domain in $R_{N}$, the assumptions of the lemma imply that for every $\mathbf{u}$ in $W_{1, p}(\Omega)^{m}$, the function $T_{h} \mathbf{u}$ is in $W_{1, r}^{\text {loc }}(\Omega)$ and inequalities (10.6), (10.7) hold a.e. in $\Omega$. This is obtained by applying the lemma in an appropriate neighborhood of each point $x$ in $\Omega$.

Using this fact, together with Sobolev's imbedding theorem, we conclude that the lemma is valid also for unbounded domains satisfying the cone condition, provided that $h(x, 0)=0$ a.e. in $\Omega$.

The lemma is essentially a consequence of [5, Theorem 2.1 and Corollary 2.1] (for the case $1<r<p<\infty$ ) and [5, Theorem 3.1] (for the case $r=p=1$ ). However some details in conditions (iii), (iv) (or(iii)', (iv)') do not formally fit in the general framework of [5]; therefore a few comments are in order. In these comments we shall refer to Theorem 2.1 of [5] which deals with the case $1<r \leqslant p<\infty$; but they apply equally well to Theorem 3.1 of [5] which deals with the case $r=p=1$.

Conditions (i) and (ii) of the lemma are stronger than the parallel conditions in [5, Theorem 2.1]. However condition (iii) (resp. (iv)) is weaker in some respect than the parallel condition I (resp. II) of [5, Theorem 2.1].

In (iii) we require that the inequality holds for every $\xi$ in a countable dense set in $R_{m}$, while in $I$ it is required that the parallel inequality holds for every $\xi$ in $R_{m}$. An examination of the proof of Theorem 2.1 of [5] reveals that condition I was used, first in order to show that $h(\cdot, \xi) \in W_{1, r}(\Omega)$ for every $\xi$ in $R_{m}$ (a fact which we assume in the lemma), and secondly in order to obtain an estimate for $\left|h\left(x^{\prime}, \xi\right)-h\left(x^{\prime \prime}, \xi\right)\right|$, namely inequality (2.19) of [5]. However, for the second application, condition I was used only with respect to a dense countable set in $\boldsymbol{R}_{m}$, as we assume here in condition (iii).

In (iv) we require that the inequality holds (for a.e. $x$ in $\Omega$ ) a.e. in $R_{m}$, while in II it is required that the parallel inequality holds (for a.e. $x$ in $\Omega$ ) at every point $\xi$ where $\partial h / \partial \xi_{i}$ exists. In the proof of Theorem 2.1 of [5] condition II 
was used in order to derive an estimate for $\left|h(x, \xi)-h\left(x, \xi^{\prime}\right)\right|$, namely inequality (2.15) of [5]. In the special case that is considered in the lemma this estimate will be

$$
\left|h(x, \xi)-h\left(x, \xi^{\prime}\right)\right|<\operatorname{const}\left(f^{*}(x)+|\xi|^{\nu}+\left|\xi^{\prime}\right|^{\prime}\right)\left|\xi-\xi^{\prime}\right|,
$$

for all $\xi, \xi^{\prime}$ in $R_{m}$ and every $x$ in $\Omega \backslash M$ (M a null set), when $p<N$;

$$
\left|h(x, \xi)-h\left(x, \xi^{\prime}\right)\right|<\text { const } f_{a}^{*}(x)\left|\xi-\xi^{\prime}\right|, \quad(\forall a>0),
$$

for all $\xi, \xi^{\prime}$ bounded in norm by $a$ and every $x$ in $\Omega \backslash M$, when $p>N$, where the constants depend only on $m, b$ and $b^{*}$. However (10.8) (resp. $(10.8)_{2}$ ) can be derived from conditions (ii) and (iv) (resp. (iv)') of the lemma, by the same argument that was used in $\$ 7$ to prove inequality (7.5).

We note that inequalities (10.6) and (10.7) follow from (10.8) (for $T_{h} \mathrm{u}$ ) and from [5, Corollary 2.1] (for $D_{x_{i}}\left(T_{h} \mathrm{u}\right)$ ). Finally the statement made in Remark 1 follows from [5, Theorem 3.3] and our previous comments here. In fact the additional assumptions mentioned in this remark are stronger than those required in [5].

Using Lemma 5 one can derive a more refined set of sufficient conditions for $T_{h}$ to map $W_{1, p}(\Omega)^{m}$ into $W_{1, r}(\Omega)$. The next theorem gives such a set of conditions.

THEOREM 8. Let $\Omega$ be a domain in $R_{N}$ satisfying the cone condition and let $p, r$ be two numbers such that either $1<r<p<\infty$ and $p \neq N$ or $r=p=1$. Suppose that $h$ is a real continuous function on $\Omega \times R_{m}$ satisfying conditions (i) and (ii) of Lemma 5. If $\Omega$ is unbounded suppose also that $h(x, 0)=0$ everywhere in $\Omega$. Further, if $p<N$, suppose that there exist

$$
\begin{aligned}
& \text { a bounded sequence }\left\{f_{n}\right\} \text { in } L_{r}(\Omega), \\
& \text { a bounded sequence }\left\{f_{n}^{*}\right\} \text { in } L_{s}(\Omega) \text { with } s=r p /(p-r), \\
& \text { an increasing sequence of open subsets of } \Omega, \text { say }\left\{\Omega_{n}\right\},
\end{aligned}
$$

such that

(A) $v \in W_{1, r}(\Omega) \Leftrightarrow v \in W_{1, r}\left(\Omega^{\prime}\right)$ where $\Omega^{\prime}=\cup_{1}^{\infty} \Omega_{n}$,

(B) $h$ satisfies conditions (iii), (iv) of Lemma 5 in every set $\Omega_{n}$, with $f$ and $f^{*}$ replaced by $f_{n}$ and $f_{n}^{*}$ and with constants $b$ and $b^{*}$ independent of $n$.

Finally, if $p>N$ or $p \geqslant 1$ and $N=1$, suppose that for every $a>0$ there exist sequences $\left\{f_{n, a}\right\}$ and $\left\{f_{n, a}^{*}\right\}$ as before such that (with $\left\{\Omega_{n}\right\}$ as before) $h$ satisfies conditions (iii)', (iv)' in every set $\Omega_{n}$ with $f_{a}, f_{a}^{*}$ replaced by $f_{n, a}, f_{n, a}^{*}$. Suppose also that condition (A) holds.

Then $T_{h}$ maps $W_{1, p}(\Omega)^{m}$ into $W_{1, r}(\Omega)$ and the mapping is bounded.

It is known that, if $r>1$ and the set $\Omega \backslash \Omega^{\prime}$ is $(1, r)$ polar and $\left(1, r^{\prime}\right)$ polar, then condition (A) holds (see e.g. [3, p. 22]). 
Proof. Let $\mathrm{u} \in W_{1, p}(\Omega)^{m}$. Applying Lemma 5 and Remark 2 to each of the sets $\Omega_{n}, n=1,2, \ldots$, we conclude that $T_{h} \mathrm{u} \in W_{1, r}^{\text {loc }}\left(\Omega^{\prime}\right)$ and that $T_{h} \mathbf{u}$ satisfies the inequalities $(10.6)$ or $(10.7)$ with $\Omega, f, f^{*} \ldots$ replaced by $\Omega_{n}, f_{n}, f_{n}^{*} \ldots$ Note that the constants appearing in these inequalities will be independent of $n$. Further, applying Sobolev's imbedding theorem to the space $W_{1, p}(\Omega)$ and using $(10.6)$ or $(10.7)$ we conclude that $T_{h} \mathbf{u} \in W_{1, r}\left(\Omega^{\prime}\right)$ and

$$
\begin{aligned}
& \left\|T_{h} \mathbf{u}\right\|_{W_{1,(}\left(\Omega^{\prime}\right)}<\operatorname{const}\left(1+\|\mathbf{u}\|_{W_{1,}(\Omega)^{m}}^{m+1}\right), \quad \text { if } p<N, \\
& \| T_{h} u_{W_{1,(}\left(\Omega^{\prime}\right)}<\operatorname{const}\left(1+\|\mathbf{u}\| W_{1,(\Omega)^{m}}\right), \\
& \text { if } p>N \text { or } p>1 \text { and } N=1 .
\end{aligned}
$$

Finally, in view of condition (A), $T_{h} \mathbf{u} \in W_{1, r}(\Omega)$.

REMARK 3. Using Remark 1 it is easily seen that the result of the theorem remains valid in the case $1=r<p<\infty$ if we assume the additional conditions mentioned in that remark.

Appendix. The purpose of this Appendix is to give a proof of Lemma 3 (\$4). First we introduce some notations. Let $k, N$ be two positive integers, $k<N$. If $x=\left(x_{1}, \ldots, x_{N}\right)$ is a point in $R_{N}$ we shall denote it also by $\left(x^{\prime}, x^{\prime \prime}\right)$ where $x^{\prime}=\left(x_{1}, \ldots, x_{k}\right)$ and $x^{\prime \prime}=\left(x_{k+1}, \ldots, x_{N}\right)$.

Lemma 3 is a consequence of the following more general result.

Proposition. Suppose that $f$ is a measurable function in $R_{N}$ such that $f\left(\cdot, x^{\prime \prime}\right) \in L_{1}^{\text {loc }}\left(R_{k}\right)$ for a.e. $x^{\prime \prime}$ in $R_{N-k}$. Let $P_{x^{\prime \prime}}$ denote the set of regular Lebesgue points of $f\left(\cdot, x^{\prime \prime}\right)$ and set $P=\cup\left\{P_{x^{n}}: x^{\prime \prime} \in R_{N-k}\right\}$. Then $P$ is a set of total measure in $R_{N}$.

REMARK. This proposition is probably known, but we do not know of a reference for it in this general form. (In the case where $f$ is the characteristic function of a measurable set, a proof can be found in [8].) Therefore we give its proof here.

Proof. For a.e. $x^{\prime \prime}$ in $R_{N-k}, P_{x^{\prime \prime}}$ is a set of total measure in $R_{k}$. Therefore, to prove the proposition we have to show only that $P$ is measurable. Given $\rho<0$ and $x$ in $R_{N}$, set

$$
F(x, \rho)=\mu_{k}\left(B^{\prime}(x, \rho)\right)^{-1} \int_{B^{\prime}(x, \rho)} f\left(y, x^{\prime \prime}\right) d \mu_{k}(y)
$$

where $B^{\prime}(x, \rho)=\left\{y \in R_{k}:\left|y-x^{\prime}\right|<\rho\right\}$. This formula can be rewritten in the form

$$
F(x, \rho)=c_{k} \int_{B_{1}^{\prime}} f\left(x^{\prime}+\rho y, x^{\prime \prime}\right) d \mu_{k}(y),
$$

where $B_{1}^{\prime}$ is the unit ball, centered at the origin, in $R_{k}$ and $c_{k}^{-1}$ is the volume 
of $B_{1}^{\prime}$. The function $\tilde{f}_{\rho}: R_{N} \times R_{k} \rightarrow R$ given by $\tilde{f}_{\rho}\left(x^{\prime}, x^{\prime \prime}, y\right)=f\left(x^{\prime}+\rho y, x^{\prime \prime}\right)$ is obviously measurable, for every $\rho$. Therefore, applying Fubini's theorem to the positive part and the negative part of $\tilde{f}_{\rho}$, we conclude that $F(\cdot, \rho)$ is measurable in $R_{N}$, for every $\rho>0$. Therefore the set

$$
Q=\left\{x \in R_{N}: f(x)=\lim _{\substack{\rho \rightarrow 0 \\ \rho \text { rational }}} F(x, \rho)\right\}
$$

is measurable. Obviously $Q \supseteq P$; but if $x^{\prime \prime}$ is a point in $R_{N-k}$ such that $f\left(\cdot, x^{\prime \prime}\right) \in L_{1}^{\text {loc }}\left(R_{k}\right)$ then $F(x, \cdot)$ is continuous for $\rho$ in $(0, \infty)$ and so

$$
Q_{x^{\prime \prime}}=\left\{x^{\prime} \in R_{k} \mid\left(x^{\prime}, x^{\prime \prime}\right) \in Q\right\}=P_{x^{\prime \prime}}
$$

Since this equality holds for a.e. $x^{\prime \prime}$ in $R_{N-k}$ it follows that $Q \backslash P$ is a null set and hence that $P$ is measurable.

\section{REFERENCES}

1. H. Federer, Geometric measure theony, Die Grundlehren der Math. Wissenschaften, Band 153, Springer-Verlag, New York, 1969.

2. E. Gagliardo, Proprieta di alcune classi di funzioni in piu variabili, Ricerche Mat. 7 (1958), 102-137.

3. J. L. Lions, Problèmes aux limites dans les equations aux dérivées partielles, Presses Univ. Montréal, Montréal, 1965.

4. M. Marcus and V. J. Mizel, Absolute continuity on tracks and mappings of Sobolev spaces, Arch. Rational Mech. Anal. 45 (1972), 294-320.

5. , Nemitsky operators on Sobolev spaces, Arch. Rational Mech. Anal. 51 (1973), 347-370.

6. __ Every superpasition operator which maps one Sobolev space into another is contimuous, J. Functional Anal. (to appear).

7. C. B. Morrey, Multiple integrals in the calculus of variations, Die Grundlehren der Math. Wissenschaften, Band 130, Springer-Verlag, Berlin and New York, 1964.

8. S. Saks, Theory of the integral, 2nd ed., Dover, New York, 1964.

9. G. Stampacchia, Equations elliptiques du second ordre à coefficients discontinus, Univ. of Montreal Press, Montreal, Quebec, 1966.

Department of Mathematics, IsRael Institute of Technology, Technon, Haifa, IsRael

Department of Mathematics, Carnegie-Mellon University, Pittsburgh, Pennsylvania 15213 